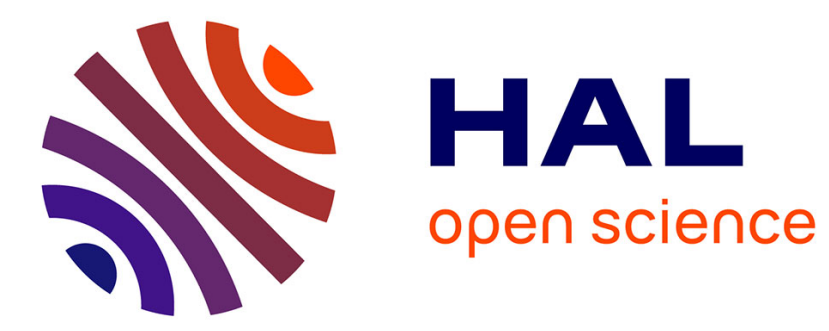

\title{
Homoclinic orbit to a center manifold
}

Patrick Bernard

\section{To cite this version:}

Patrick Bernard. Homoclinic orbit to a center manifold. Calculus of Variations and Partial Differential Equations, 2003, 17 (2), pp.121-157. 10.1007/s00526-002-0162-0 . hal-01251761

\section{HAL Id: hal-01251761 https://hal.science/hal-01251761}

Submitted on 6 Jan 2016

HAL is a multi-disciplinary open access archive for the deposit and dissemination of scientific research documents, whether they are published or not. The documents may come from teaching and research institutions in France or abroad, or from public or private research centers.
L'archive ouverte pluridisciplinaire HAL, est destinée au dépôt et à la diffusion de documents scientifiques de niveau recherche, publiés ou non, émanant des établissements d'enseignement et de recherche français ou étrangers, des laboratoires publics ou privés. 
Patrick Bernard

\section{Homoclinic orbit to a center manifold}

Received: 11 Februay 2001 / Accepted: 23 January 2002 /

Published online: 6 August 2002 - (c) Springer-Verlag 2002

\section{Introduction}

A saddle-center fixed point of a Hamiltonian system is a fixed point with precisely one pair of purely imaginary eigenvalues, and other eigenvalues all having non-zero real part. Such a fixed point is contained in a two dimensional invariant manifold, called the center manifold, associated with the pair of imaginary eigenvalues and filled with periodic orbits. Each of these periodic orbits is the transversal intersection between its energy shell and the center manifold, and is hyperbolic with respect to its energy shell. We are interested in the existence of orbits homoclinic to these periodic trajectories.

Let us consider an initial system with a saddle-center fixed point and an orbit homoclinic to it. The orbit structure near this homoclinic orbit of the initial system and of perturbed systems can be studied using appropriate local sections and the Poincaré return map along the homoclinic. This has been initiated by Conley in [13], and used in [23], [25] and [28] to prove the existence of homoclinic orbits in perturbed systems. Under suitable hypotheses, and if the phase space is four dimensional, these papers show the following behavior. Hamiltonian systems sufficiently close to the initial system have a saddle-center fixed point with a center manifold. We can suppose without loss of generality that the saddle-center fixed point always has zero energy in the systems under interest. For any sufficiently small fixed positive energy, the periodic motion on the center manifold at that energy has a homoclinic orbit in a system sufficiently close to the initial system. However, in a fixed system close to the initial system, the periodic orbits closest to the fixed point are not proved to have any homoclinic orbit. The homoclinic orbits of smallest energy are first destroyed by the perturbation. This is not surprising since the saddle-center fixed point itself does not have any homoclinic orbit in general. These works provide a much more detailed description of the orbit structure than we do in this paper, but their range is limited to the study of perturbations of initial systems

P. Bernard: Institut Fourier, Université Grenoble I, BP 74, 38402 Saint Martin d'Hères cedex, France (e-mail: Patrick.Bernard@ujf-grenoble.fr) 
with a homoclinic orbit to the saddle-center, which is an exceptional case, and to four dimensional phase spaces.

Variational methods provide global existence results on homoclinic orbits to a hyperbolic fixed point, see [6], [14] and many other papers, that can be viewed as non-perturbative analogs of the theory of Melnikov that studies the persistence of homoclinics under perturbation. In the same spirit, we attempt to provide a nonperturbative analog of the behavior described above around saddle-center fixed points. This paper is closely connected to [4], where we study the existence of homoclinic orbits to some hyperbolic periodic orbits of a Hamiltonian system in $\mathbb{C}^{n}$. Since the smallest periodic motion in the center manifold having a homoclinic orbit seems to go away from the fixed point when the system goes away from the initial system in the perturbative setting, it is natural to consider a global center manifold in order to find a global (i.e. non-perturbative) result. In [4], we studied the vicinity of a prescribed energy shell sufficiently far from the origin, and obtained homoclinic orbits in a dense family of energy shells around the prescribed one. We abandon all pretension to find many homoclinic orbits, but focus on finding the orbit closest to the saddle-center. It is yet very unlikely that the orbit we find is indeed the closest to the saddle-center, but it is probably the closest among those which satisfy a certain estimate. We shall clarify this point later.

We study a model system where the center manifold is a plane with harmonic oscillations on it. We suppose that these periodic motions are hyperbolic with respect to their energy shells, so that the center manifold is a normally hyperbolic manifold. The setting is thus quite similar to the setting of [4], but we assume here that the total phase space is the product of this plane with the cotangent bundle of a compact manifold $M$, instead of $\mathbb{R}^{2 n}$ in [4]. This product structure is a key to our result, since we shall obtain homoclinic orbits by comparison with product (uncoupled) flows. The existence of homoclinic orbits for product flows is reduced to well-known existence results on homoclinic motions to hyperbolic fixed points on compact Riemannian manifolds, see [6]. We shall moreover assume that the Hamiltonian is fiberwise convex on the total phase space $T^{*}(M \times \mathbb{R})$, so that a Lagrangian action functional can be used. It should be possible to avoid this restriction since the Hamiltonian action functional can be well studied in this context, see [20] or [11]. Yet the Lagrangian functional remains simpler, and the results of this paper will be expressed in terms of Lagrangian systems.

Let us stress that, although the setting is Lagrangian, our result is very different from classical ones on homoclinic orbits in Lagrangian systems since the periodic orbits of the center manifold do not satisfy the minimality hypothesis needed in these results. In fact, the center manifold as a whole satisfies this hypothesis, and we shall look for orbits homoclinic to this manifold. An orbit homoclinic to the center manifold is homoclinic to one of the periodic orbits, by energy conservation. The difficulty is that the center manifold is not compact, and that we have to find a way to localize orbits.

Under suitable hypotheses, we prove the existence of an orbit homoclinic to one of the oscillations of the center manifold and give an estimate of its action and of its energy. These estimates are the main novelties compared with [4], they allow interesting new applications. The energy is close to zero (the energy of the 

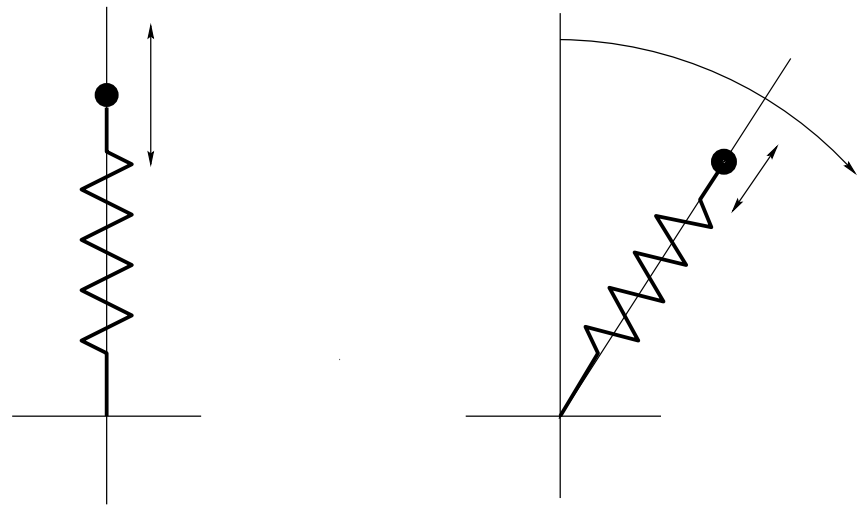

Fig. 1. Elastic pendulum

fixed point) when the system is close to a product system and the homoclinic we find should be seen as the continuation, when a coupling is introduced, of the orbit homoclinic to the origin that existed in the product system. In this sense we can say that the homoclinic we find is the closest to the origin, although new orbits, longer and closer, may appear. Both the center manifold and the homoclinic orbit are preserved by a small perturbation of the system i.e. a perturbed system still has an invariant manifold diffeomorphic to a plane and foliated by periodic orbits one of which has a homoclinic.

Among the applications let us give the example of the stiff elastic spatial pendulum. This is a pendulum where the bar has been replaced by a stiff spring which has variable length but remains always straight, see Fig. 1. The center manifold here is the set of oscillations of the spring in unstable equilibrium. We obtain an orbit homoclinic to one of these oscillations when the spring is stiff enough. This homoclinic is moreover preserved by a small perturbation of the system. It is a very general process to introduce an additional degree of freedom highly confined to zero in a mechanical system (a previously frozen binding is now granted some freedom to oscillate). Under certain hypotheses, we see that a hyperbolic fixed point with a homoclinic orbit of the frozen system is turned to a saddle-center fixed point with a center manifold and an orbit homoclinic to the center manifold in the extended system.

A major interest of homoclinic orbits is their link with chaotic behavior. The orbit structure near a transversal homoclinic orbit to a hyperbolic fixed point of a periodic time-dependent system has by now been well described. The natural analog of this structure exists in an autonomous system around a transversal homoclinic orbit to a hyperbolic periodic orbit. It should be noted however that the behavior associated with homoclinic orbits to hyperbolic fixed points of autonomous systems is not as well understood, see [16] and [9] for some results on this subject. One of the interests of our work is that the homoclinic we find, if transversal, lead to the well described case, i.e. to a Bernoulli shift with topological entropy. Consider for example a classical plane pendulum, our results provide a new way to break integrability and introduce chaotic behavior. Instead of considering that there is 
some small influence from the exterior (a time dependent perturbation), one can consider that the bar has some elasticity. In this case, the unstable equilibrium is surrounded by unstable oscillations. We prove that one of these oscillations have a homoclinic orbit, this homoclinic can be made transversal by a perturbation, and the system then has topological entropy.

The questions discussed in this paper were asked to me by my advisor, Eric Séré. It is a pleasure to acknowledge his decisive helps and encouragements. I also wish to thank Ivar Ekeland for his interesting comments.

\section{Results, comments and applications}

Let $M$ be a compact manifold, $T M \stackrel{\pi}{\longrightarrow} M$ its tangent bundle. We provide $M$ with a metric $g$, and note

$$
\|z\|=\sqrt{g_{\pi(z)}(z, z)}
$$

the norm of a tangent vector $z \in T M$. There is an associated metric on $T M$, and we note $d\left(z, z^{\prime}\right)$ the distance between two points of $T M$ associated with this metric. Let us consider the smooth Lagrangian on $T(M \times \mathbb{R})=T M \times \mathbb{R}^{2}$ given by

$$
L(z, q, v)=a\left(v^{2}-\omega^{2} q^{2}\right)+G(z, q, v) \quad(z, q, v) \in T M \times \mathbb{R} \times \mathbb{R},
$$

where $a$ and $\omega$ are positive real numbers and $G: T M \times \mathbb{R}^{2} \longrightarrow \mathbb{R}$ is a smooth function satisfying the assumptions:

HG1 : There exists a $z_{0}=\left(\theta_{0}, 0\right) \in T M$ such that $G\left(z_{0}, q, v\right)=0$ and $d G\left(z_{0}, q, v\right)=0$ for all $(q, v) \in \mathbb{R}^{2}$.

HG2 : There exists a $b>0$ such that $G(z, q, v) \geqslant b d\left(z, z_{0}\right)^{2}$.

HG3 :

$$
G(z, q, v) \geqslant \frac{1}{2}\left(q \frac{\partial G}{\partial q}(z, q, v)+v \frac{\partial G}{\partial v}(z, q, v)\right)+b d\left(z, z_{0}\right)^{2} .
$$

Moreover, we assume that there exist two smooth fiberwise convex functions $U$ and $W$ on $T M$ such that

$$
U(z) \leqslant G(z, q, v) \leqslant W(z)
$$

for all $(z, q, v) \in T M \times \mathbb{R}^{2}$, and that they both satisfy

HU1 : $U\left(z_{0}\right)=0$ and $d U\left(z_{0}\right)=0$,

HU2: $U(z) \geqslant b d\left(z, z_{0}\right)^{2}$.

Finally, we assume that the Lagrangian $L$ is fiberwise convex,

HL : The restriction of $L$ to each fiber $T_{\theta} M \times T_{q} \mathbb{R}$ is convex, with uniformly positive Hessian.

No more control at infinity is necessary for our results to hold true, but we will use in the proofs systems satisfying the additional hypothesis

HG4 : There exists a function $G_{\infty}$ on $T M$, a number $\alpha>0$, a compact set $K \subset T M$ and a compact set $B \subset K \times \mathbb{R}^{2}$ such that $G(z, q, v)=G_{\infty}(z)$ outside $B$, and $G_{\infty}(z)=\alpha\|z\|^{2}$ outside $K$.

As a consequence of [HL], the trajectories of $L$ on $M \times \mathbb{R}$ are the projections of 
the integral curves of a vector-field $Y_{L}$ on $T M$. This vector-field is conjugated to the Hamiltonian vector field $X_{H}$ on $T^{*} M$, where $H$ is the fiberwise dual of $L$

$$
H(\zeta, q, p)=\sup _{z \in \pi^{-1}\left(\pi^{*}(\zeta)\right), v \in \mathbb{R}}\langle\zeta, z\rangle+p v-L(z, q, v) \quad(\zeta, q, p) \in T^{*} M \times \mathbb{R}^{2}
$$

See Section 2 for more details. The flow of $Y_{L}$ has an invariant manifold, the center manifold, of equation $z=z_{0}$. The center manifold is filled with periodic orbits, which are the liftings of

$$
O_{r}(t)=\left(\theta_{0}, r \cos (\omega t)\right),
$$

and can be described also by

$$
O_{r}=\left\{\left(z_{0}, q, v\right) \in T M \times \mathbb{R}^{2} / v^{2}+\omega^{2} q^{2}=\omega^{2} r^{2}\right\} .
$$

We are looking for orbits homoclinic to $O_{r}$, i.e. trajectories $x=(\theta, q): \mathbb{R} \longrightarrow$ $M \times \mathbb{R}$ such that $\theta \not \equiv \theta_{0}$ and

$$
\lim _{t \rightarrow \pm \infty}\left(\theta(t), \dot{\theta}(t), \dot{q}(t)^{2}+\omega^{2} q(t)^{2}\right)=\left(\theta_{0}, 0, \omega^{2} r^{2}\right) .
$$

We will see in Section 5 that to any function $U$ on $T M$ satisfying [HU1,2] we can associate a number $\mathrm{I}(\mathrm{U})$ such that

$$
U \leqslant W \Longrightarrow I(U) \leqslant I(W)
$$

and

$$
\left|1-\frac{I(W)}{I(U)}\right| \leqslant \sup _{z} \frac{|W(z)-U(z)|}{b d^{2}\left(z, z_{0}\right)}
$$

for all $U$ and $W$ satisfying [HU1-2]. Recall that $b$ is the constant of [HU2]. The value $I(U)$ can be thought of as the action of an orbit homoclinic to $z_{0}$ for the Lagrangian system $U$ on $T M$, although we can only prove that there is a homoclinic of action below $I(U)$. We are now in a position to state the main result of this paper:

Theorem 1 Let us consider the Lagrangian system (1), and assume that G satisfies [HG1-3], [HL], and

$$
U(z) \leqslant G(z, q, v) \leqslant W(z)
$$

with $U$ and $W$ satisfying [HU1,2]. There is a radius

$$
r \leqslant \sqrt{\frac{I(W)-I(U)}{2 \pi a \omega}}
$$

such that the periodic orbit $O_{r}$ has a homoclinic orbit $X_{\infty}=\left(\theta_{\infty}, q_{\infty}\right)$. This orbit moreover satisfies

$$
\int_{\mathbb{R}} G\left(\partial X_{\infty}\right)-\frac{1}{2} q_{\infty} \frac{\partial G}{\partial q}\left(\partial X_{\infty}\right)-\frac{1}{2} \dot{q}_{\infty} \frac{\partial G}{\partial v}\left(\partial X_{\infty}\right) \leqslant I(W) .
$$


In the expression above, $\partial X$ is the lifting of $X$, see Section 2. This paper is organized as follows. First we comment the theorem, and give some applications in the next subsections. In Section 2, we recall some general facts about Hamiltonian and Lagrangian systems. These facts will be used throughout the paper. The detailed analysis of the local behavior of the flow in Section 6 may be of independent interest, while section 5 provides a concise account about the existence of homoclinic orbits in Lagrangian Systems of the kind $U$ on $T M$, and gives the precise definition of the number $I(U)$. The proof of Theorem 1 is explained in Section 3, and detailed in the last sections of the paper. We show in Section 4 how to change the Lagrangian function at infinity in order to be reduced to a Lagrangian satisfying [HG4].

\section{Remarks.}

1. A very similar result is obtained in [4]. Beyond the fact that the setting is different, the main interest of the present result is that we obtain an explicit estimate of the maximum radius (4), which, combined with (3), allows in certain instances to prove that the homoclinic we find is actually close to the saddlecenter. This enables new applications. The estimate (5) is also new, we have to relax it in [4] to localize the homoclinic orbits. Our belief is that the homoclinic we obtain is the closest to the fixed point among those which satisfy (5). The price for these estimates is that we obtain only one homoclinic orbit, while infinitely many are found in [4]. It should be possible, although not so easy, to carry over the results of this paper to the setting of [4], and the results of [4] to this setting.

2. As a consequence of the hypotheses [HG1,2], the orbit $O_{r}$ is hyperbolic with respect to its energy shell and the fixed point $\left(\theta_{0}, 0\right)$ is of saddle center type, with $2 n$ hyperbolic dimensions and 2 elliptic dimensions in phase space. This is proved in Section 6.

3. The hypothesis [HG3] can also be written

$$
L(z, q, v) \geqslant \frac{1}{2}\left(q \frac{\partial L}{\partial q}(z, q, v)+v \frac{\partial L}{\partial v}(z, q, v)\right)+c d\left(z, z_{0}\right)^{2}
$$

or in the Hamiltonian form

$$
H(\zeta, q, p)+c d^{2}\left(H_{\nu}, z_{0}\right) \leqslant \frac{1}{2}\left(q \frac{\partial H}{\partial q}+p \frac{\partial H}{\partial p}\right)+\left\langle\zeta, H_{\nu}\right\rangle,
$$

where $H_{\nu} \in T M$ is the derivative of $H$ with respect to the fiber to $T^{*} M$.

4. Let $E$ be the energy, see Section 2. It can be easily computed (see Section 6) that

$$
E\left(O_{r}\right)=a \omega^{2} r^{2}
$$

and the energy of the homoclinic obtained from Theorem 1 satisfies

$$
0 \leqslant E\left(\partial X_{\infty}\right) \leqslant E_{0}=\frac{\omega}{2 \pi}(I(W)-I(U))
$$


5. The integral $\int_{\mathbb{R}} L(\partial X)$ is not defined for a homoclinic orbit because it has an oscillating tail. This is linked to the fact that the action

$$
\int_{0}^{t} L\left(\partial O_{r}(s)\right) d s
$$

is not identically zero. We can nevertheless integrate by parts the expression

$$
\int \dot{q}^{2}-\omega^{2} q^{2}=[q \dot{q}]-\int q\left(\ddot{q}+\omega^{2} q\right),
$$

and using the Euler-Lagrange equation

$$
2 a\left(\ddot{q}+\omega^{2} q\right)=\frac{\partial G}{\partial q}-\frac{d}{d t}\left(\frac{\partial G}{\partial v}\right),
$$

and a second integration by parts we obtain

$$
\mathcal{L}\left(X_{\infty}\right)=a\left[q_{\infty} \dot{q}_{\infty}\right]+\int_{\mathbb{R}} G\left(\partial X_{\infty}\right)-\frac{1}{2} q_{\infty} \frac{\partial G}{\partial q}\left(\partial X_{\infty}\right)-\frac{1}{2} \dot{q}_{\infty} \frac{\partial G}{\partial v}\left(\partial X_{\infty}\right),
$$

thus the integral can be thought as the action of the homoclinic orbit.

6. Although the setting is Lagrangian, the theory of Bolotin [7] can not be applied to our problem. Here the whole center manifold enjoys a minimizing property as used in [7], but the orbits $O_{r}$ themselves do not. This is connected with the fact that the center manifold is hyperbolic with respect to the full phase space, while the periodic orbit $O_{r}$ is not. For that reason, we shall rather search orbits homoclinic to the center manifold as a whole, and this is why we do not know precisely which of the periodic orbits $O_{r}$ have a homoclinic.

7. It should be possible to extend Theorem 1 to more general Hamiltonian systems by using the analysis of [20] or pseudo-holomorphic curves as in [10] and [11], [12].

\subsection{Normalization of the center manifold and persistence of the hypotheses}

The hypotheses of Theorem 1 may appear to be very rigid. They imply for example that there is an invariant plane with elliptic linear motion on it. We see in this section a general method for normalizing center manifolds i.e. bringing them to a linear elliptic plane. This requires a change of coordinates and a reparametrisation. These operations preserve homoclinic orbits. This method can be applied to prove that the homoclinic obtained by Theorem 1 is not destroyed by a $C^{3}$-small perturbation of $L$.

Theorem 2 Let L be a Lagrangian function satisfying all the hypotheses of Theorem 1, let

$$
E_{0}>\frac{\omega}{2 \pi}(I(W)-I(U))
$$

be a fixed energy and let $K$ be a compact set of $T M \times \mathbb{R}^{2}$ containing $\left\{E \leqslant E_{0}+1\right\}$. There is a $\epsilon>0$ such that any perturbed Lagrangian $L_{\epsilon}$ satisfying $\left\|L_{\epsilon}-L\right\|_{C^{3}(K)} \leqslant$ $\epsilon$ has a saddle-center fixed point $p(\epsilon)$ and a center manifold $\mathcal{C}(\epsilon)$ intersecting each 
energy shell $\{E=e\}, E_{\epsilon}(p(\epsilon))<e \leqslant E_{0}$ transversally along a closed integral curve of the associated vector field $Y_{\epsilon}$. Each of these periodic orbits

$$
\mathcal{C}(\epsilon) \cap\{E=e\}, \quad E_{\epsilon}(p(\epsilon)) \leqslant e \leqslant E_{0}
$$

is moreover hyperbolic with respect to its energy shell, and one of them has a homoclinic orbit.

Let us start with some general comments before we prove Theorem 2 . We call a non-degenerate fixed point $p$ of a Hamiltonian vector field on a $2 n+2$-dimensional symplectic manifold a saddle-center if the linearized vector field at $p$ has one pair of purely imaginary eigenvalues $\pm i \omega$ and if the $2 n$ other eigenvalues have nonzero real part. By a theorem of Lyapunov, there exists a unique local center manifold, which is an invariant two dimensional symplectic manifold. There are symplectic coordinates $\left(x_{i}, y_{i}\right)_{0 \leqslant i \leqslant n}$ around $p$ such that the local center manifold is a neighborhood of the origin in the plane $\left(x_{0}, y_{0}\right)$. The induced flow on this two-dimensional plane is integrable, and we can choose the coordinates $\left(x_{0}, y_{0}\right)$ such that the induced Hamiltonian is $H\left(x_{0}, y_{0}, 0, \ldots, 0\right)= \pm f\left(x_{0}^{2}+y_{0}^{2}\right)$, with a smooth function $f$ such that $f^{\prime}(0)=\omega>0$. There is an increasing function $g:\left(-\infty, h_{0}\right] \longrightarrow \mathbb{R}$ such that $g=f$ on an interval $\left[0, h_{0}\right]$, with some $h_{0}>0$. The Hamiltonian function $\tilde{H}=g^{-1}( \pm H / 2)$ is defined on $\left\{ \pm H \leqslant \pm 2 f\left(h_{0}\right)\right\}$. The point $p$ is a saddlecenter fixed point of $\tilde{H}$, its center manifold is the plane $\left(x_{0}, y_{0}\right)$ in local charts, and $\tilde{H}\left(x_{0}, y_{0}, 0, \ldots, 0\right)=\left(x_{0}^{2}+y_{0}^{2}\right) / 2$ when $x_{0}^{2}+y_{0}^{2} \leqslant h_{0}$. We say that $\tilde{H}$ has a normalized center manifold. The important point is that there is a homoclinic for $H$ if there is a homoclinic for $\tilde{H}$. Such a homoclinic may be found under additional hypotheses by applying Theorem 1 to a Hamiltonian system extending $\tilde{H}$. This can be done for example when $H$ is a perturbation of a system satisfying the hypotheses of Theorem 1. Let us now focus our attention on this situation.

The center manifold is globally preserved by a perturbation. To make this precise, let us consider a Lagrangian given by (1), satisfying [HL] and [HG1,2], and a one parameter family of Lagrangians $L_{\epsilon}$ satisfying

$$
\left\|L_{\epsilon}-L\right\|_{C^{3}} \leqslant \epsilon
$$

and such that $L_{\epsilon}-L=0$ outside some fixed compact subset $K$ of $T M \times \mathbb{R}^{2}$. The associated Hamiltonian function $H_{\epsilon}$ satisfies $\left\|H_{\epsilon}-H\right\|_{C^{3}} \leqslant o_{\epsilon}(1)$ and $H_{\epsilon}-H=0$ outside $K$. The periodic orbits filling $\left(\theta_{0}, 0\right) \times \mathbb{R}^{2}$ for the unperturbed system $L$ are hyperbolic, this is proved in Section 6 . The persistence of the invariant manifold can be seen as a particularly simple case of the theory of normally hyperbolic manifolds in the sense of [19] or [18], or proved directly since the persistence of a given periodic orbit can be reduced to the persistence of a hyperbolic fixed point after taking a section and restricting to the energy shell. The perturbed manifold is smooth, and can be redressed by a global symplectomorphism. This is carried out in details in [5], where we prove the following: There is a family of compactly supported symplectic diffeomorphisms $\Phi_{\epsilon}$ with $\left\|\Phi_{\epsilon}-i d\right\|_{C^{2}}=o_{\epsilon}(1)$ and a family $f_{\epsilon}$ of functions with $\left\|f_{\epsilon}-i d\right\|_{C^{2}}=o_{\epsilon}(1)$ such that the manifold $\Phi_{\epsilon}\left(\left(\theta_{0}, 0\right) \times \mathbb{R}^{2}\right)$ is invariant for $H_{\epsilon}$, and

$$
f_{\epsilon} \circ H_{\epsilon} \circ \Phi_{\epsilon}\left(\theta_{0}, 0, q, p\right)=H_{0}\left(\theta_{0}, 0, q, p\right) .
$$


We define the normalized Hamiltonian $\tilde{H}_{\epsilon}=f_{\epsilon} \circ H_{\epsilon} \circ \Phi_{\epsilon}$, the associated Lagrangian $\tilde{L}_{\epsilon}$ can be written as (1) with a function $\tilde{G}_{\epsilon}$ satisfying [HG1], we have globally normalized the center manifold. Let us now compute

$$
\begin{aligned}
d^{2} \tilde{H}_{\epsilon}(x) \cdot(u, u) & =f_{\epsilon}^{\prime}\left(H_{\epsilon} \circ \Phi_{\epsilon}(x)\right) d^{2} H_{\epsilon}\left(\Phi_{\epsilon}(x)\right)\left(d \Phi_{\epsilon}(x) \cdot u, d \Phi_{\epsilon}(x) \cdot u\right) \\
& +f_{\epsilon}^{\prime}\left(H_{\epsilon} \circ \Phi_{\epsilon}(x)\right) d H_{\epsilon}\left(\Phi_{\epsilon}(x)\right) \circ d^{2} \Phi_{\epsilon}(x) \cdot(u, u) \\
& +f_{\epsilon}^{\prime \prime}\left(H_{\epsilon} \circ \Phi_{\epsilon}(x)\right)\left(d H_{\epsilon}\left(\Phi_{\epsilon}(x)\right) \circ d \Phi_{\epsilon}(x) \cdot u\right)^{2} .
\end{aligned}
$$

We obtain that

$$
\left\|\tilde{H}_{\epsilon}-H\right\|_{C^{2}} \underset{\epsilon \rightarrow 0}{\longrightarrow} 0
$$

which implies that

$$
\left\|\tilde{L}_{\epsilon}-L\right\|_{C^{2}} \underset{\epsilon \rightarrow 0}{\longrightarrow} 0
$$

and we also easily see that

$$
\tilde{L}_{\epsilon}-L=0 \quad \text { outside } K \text {. }
$$

We use this normalized form to prove Theorem 2.

Proof of Theorem 2. The first step is to replace the Lagrangian $L_{\epsilon}$ by a new Lagrangian, still noted $L_{\epsilon}$, which satisfies

$$
\left\|L_{\epsilon}-L\right\|_{C^{3}} \leqslant C_{K} \epsilon
$$

and such that $L_{\epsilon}=L$ outside $K$. This can be done with a constant $C_{K}$ depending only on $K$. When $\epsilon$ is small enough, the associated vector field has a global center manifold. We now consider the normalized Lagrangian

$$
\tilde{L}_{\epsilon}=a\left(v^{2}-\omega^{2} q^{2}\right)+\tilde{G}_{\epsilon}(z, q, v) \quad(z, q, v) \in T M \times \mathbb{R} \times \mathbb{R},
$$

as defined above. Let us stress that $L_{\epsilon}$ has an orbit homoclinic to a periodic trajectory $\mathcal{C}(\epsilon) \cap\{E=e\}$ for some $e \in\left[E_{\epsilon}(p(\epsilon)), E_{0}\right]$ if $\tilde{L}_{\epsilon}$ has an orbit homoclinic to a periodic trajectory $O_{r}$ for some $r$ satisfying $a \omega r^{2} \leqslant E_{0}$. We apply Theorem 1 to find such a homoclinic orbit. There remains to check that the hypotheses of Theorem 1 are satisfied by $\tilde{L}_{\epsilon}$. The Lagrangian $\tilde{L}_{\epsilon}$ has been constructed to obtain [HG1]. The hypothesis [HL] is a direct consequence of (6) and (7) when $\epsilon$ is small enough. It is not harder to see that [HG2] holds with the constant $b / 2$ instead of $b$ for sufficiently small $\epsilon$. We also obtain from (6) and (7) the existence of a function $c(\epsilon)>0$ with $\lim _{\epsilon \rightarrow 0} c(\epsilon)=0$ such that

$$
\left|\tilde{L}_{\epsilon}-L\right| \leqslant c(\epsilon) d^{2}\left(z, z_{0}\right)
$$

and

$$
\begin{gathered}
\left|\left(q \frac{\partial L}{\partial q}(z, q, v)+v \frac{\partial L}{\partial v}(z, q, v)\right)-\left(q \frac{\partial \tilde{L}_{\epsilon}}{\partial q}(z, q, v) v \frac{\partial \tilde{L}_{\epsilon}}{\partial v}(z, q, v)\right)\right| \\
\leqslant c(\epsilon) d^{2}\left(z, z_{0}\right) .
\end{gathered}
$$


The hypothesis [HG3] is thus satisfied with the constant $b / 2$ when $\epsilon$ is small enough. We moreover have the inequality

$$
U_{\epsilon}=U-c(\epsilon) d^{2}\left(z, z_{0}\right) \leqslant \tilde{G}_{\epsilon} \leqslant W+c(\epsilon) d^{2}\left(z, z_{0}\right)=W_{\epsilon} .
$$

The estimate (3) now yields

$$
\left|I\left(W_{\epsilon}\right)-I\left(U_{\epsilon}\right)\right| \underset{\epsilon \rightarrow 0}{\longrightarrow}|I(W)-I(U)| .
$$

It is possible to apply Theorem 1 to $\tilde{L}_{\epsilon}$ when $\epsilon$ is small enough, and get a homoclinic orbit to $O_{r}$ with

$$
a \omega^{2} r^{2} \leqslant \frac{\omega}{2 \pi}\left(I\left(W_{\epsilon}\right)-I\left(U_{\epsilon}\right)\right) \leqslant E_{0} .
$$

\subsection{Perturbation from product systems}

Let us first consider the case where $G$ does not depend on $(q, v)$. We can set $U=G=W$ in the notations of Theorem 1 , and

$$
Q(q, v)=a\left(v^{2}-\omega^{2} q^{2}\right), \quad(q, v) \in \mathbb{R}^{2} .
$$

The system $L$ is the uncoupled product between the linear oscillating Lagrangian system $Q$ on $\mathbb{R}$ and the Lagrangian system $U$ on $M$. It is well known that if [HU1,2] hold the Lagrangian system $U$ has an orbit $h(t)$ homoclinic to $\theta_{0}$, see Section 5 . This can be recovered from Theorem 1. The hypothesis [HG3] always holds in this case, and Theorem 1 gives the existence of an orbit homoclinic to $\left(\theta_{0}, 0\right)$ for $L$, which of course implies the existence of the homoclinic of $U$. All the orbits $O_{r}$ have a homoclinic for $L$ in this case, given by

$$
h_{r}(t)=(h(t), r \cos (\omega t)) .
$$

Let us now come back to the general case of a function $G(z, q, v)$. The theorem 1 can be seen as a perturbation result when a coupling is introduced in a product as above. Elementary dimension considerations show that the saddle-center fixed point do not have any homoclinic orbit in a generic coupled system. The theorem 1 yet gives the existence of an orbit homoclinic to some periodic orbit $O_{r}$ if [HG3] holds. In view of the estimate (3) the quantity $I(W)-I(U)$ is a measure of the coupling, and we obtain that the radius $r$ tends to zero when the coupling tends to 0 . The orbit obtained by Theorem 1 can be considered as the continuation of the orbit homoclinic to the fixed point that existed in the uncoupled system. Moreover, the hypothesis [HG3] is satisfied when the coupling is small since it can be written

$$
\frac{1}{2}\left(q \frac{\partial C}{\partial q}+v \frac{\partial C}{\partial v}\right)-C \leqslant U-c d^{2}\left(z, z_{0}\right)
$$

if we separate the main part $U$ and the coupling perturbation $C$ of $R: G(z, q, v)=$ $U(z)+C(z, q, v)$, and it is satisfied for example when

$$
|C|+\left|q \frac{\partial C}{\partial q}\right|+\left|v \frac{\partial C}{\partial v}\right| \leqslant \epsilon d^{2}\left(z, z_{0}\right)
$$


with a sufficiently small $\epsilon$. Shortly, The homoclinic orbit to the fixed point that existed in the uncoupled system is turned to an orbit homoclinic to $O_{r}$ when the coupling is introduced, with $r$ as small as the coupling is small, and this homoclinic exists as long as [HG3] holds. Combining this with the methods of the preceding subsection, we obtain

Application 1 Let us consider a smooth one parameter family $L_{\epsilon}$ of Lagrangian systems such that

$$
L_{0}(z, q, v)=a\left(v^{2}-\omega^{2} q^{2}\right)+U(z), \quad(z, q, v) \in T M \times \mathbb{R} \times \mathbb{R}
$$

satisfies [HL] and [HU1,2]. There is an $\epsilon_{0}>0$ and a function $e(\epsilon) \geqslant 0$ satisfying $\lim _{\epsilon \rightarrow 0} e(\epsilon)=0$ such that for $\epsilon \leqslant \epsilon_{0}$ the system $L_{\epsilon}$ has a saddle-center fixed point $p(\epsilon)$ and a center manifold $\mathcal{C}(\epsilon)$ intersecting transversally the energy level $E_{\epsilon}^{-1}(e(\epsilon))$ along a hyperbolic periodic trajectory which has a homoclinic orbit.

\subsection{Singular perturbation}

The case $\omega \longrightarrow \infty$ is of physical interest. Let us consider a system

$$
L^{\omega}(z, q, v)=a\left(v^{2}-\omega^{2} q^{2}\right)+G(z, q), \quad(z, q, v) \in T M \times \mathbb{R} \times \mathbb{R},
$$

and set

$$
G_{0}(z)=G(z, 0), \quad z \in T M .
$$

When $\omega$ is large, the term $a \omega^{2} q^{2}$ in $L$ can be seen as a potential confining the system on the subspace $M \times\{0\}$ of the total configuration space $M \times \mathbb{R}$. Taking $\omega \longrightarrow \infty$ approximates the case of a holonomic constraint, see [1], chapter 4, or [2], p 41. At the limit, the configuration of the system is forced to stay in $M=M \times\{0\}$, and its evolution is described by the Lagrangian flow of $G_{0}$ on $T M$. Let us suppose that there is a critical point $z_{0}=\left(\theta_{0}, 0\right) \in T M$ of $G_{0}$ such that $G_{0}\left(z_{0}\right)=0$ and

HG2 : There is a $b>0$ such that $G_{0}(z) \geqslant b d^{2}\left(z, z_{0}\right)$.

The point $z_{0}=\left(\theta_{0}, 0\right)$ is then a hyperbolic rest point of the limit flow (the flow of $G_{0}$ ) and there is an orbit of $G_{0}$ homoclinic to this fixed point, see Section 5. It is interesting to study the limit process and describe what remains of this homoclinic orbit in the total flow for large but finite $\omega$. We will furthermore assume that $G$ satisfies

HG1 loc : There exist an $\epsilon>0$ and a continuous function $C(q)$ such that, when $|q| \leqslant \epsilon, G\left(z_{0}, q\right)=0, d G\left(z_{0}, q\right)=0$ and

$$
\left|\frac{\partial G}{\partial q}\right| \leqslant C(q) d^{2}\left(z, z_{0}\right)
$$

for all $z$.

Example. Let us consider a pendulum, in the plane or in space, where the bar is replaced by a stiff spring which has variable length but remains always straight, see Fig. 1, page 123. The Lagrangian of this system can be written

$$
L(\theta, \dot{\theta}, q, \dot{q})=\dot{q}^{2}-\omega^{2} q^{2}+\left(l_{0}+q\right)^{2} \dot{\theta}^{2}+\left(l_{0}+q\right)(1-\cos \varphi(\theta))
$$


where $\theta \in S^{2}$ is the direction of the spring, $\varphi(\theta)$ is the angle between the spring and the vertical axis pointing up, and $l_{0}+q$ is the length of the spring, $l_{0}$ being its length in the unstable equilibrium position. Let us call $\theta_{0}$ the vertical direction pointing up, that is the direction of the unstable equilibrium. It is not hard to check that both hypotheses above hold for that system. There is an unstable invariant manifold $(\theta, \dot{\theta})=\left(\theta_{0}, 0\right)$ filled with oscillations of the spring. In view of the application below, one of these oscillations has a homoclinic orbit if the spring is stiff enough. The whole structure, center manifold and homoclinic orbit, is preserved by a small perturbation. The homoclinic of the stiff elastic pendulum can be seen as the continuation of the homoclinic that exists in the rigid pendulum, which is the limit system when the stiffness tends to infinity. Note that the energy of the homoclinic orbit does not tend to zero in general when the stiffness tends to infinity (or at least we can not prove that it does) although the length of the spring is converging to $l_{0}$. The homoclinic has small but fast oscillations.

Application 2 Let us consider a Lagrangian $L^{\omega}$ as defined above, satisfying the hypotheses [HG2] and [HG1 loc]. The point $\left(z_{0}, 0,0\right)$ is a saddle-center fixed point of $L^{\omega}$. It has a center manifold $z=z_{0}$, which is filled with the periodic orbits $O_{r}^{\omega}=\left\{v^{2}+\omega^{2} q^{2}=\omega^{2} r^{2}\right\}$. There is an energy $E_{\infty} \geqslant 0$ such that

- When $\omega$ is large enough there is an orbit $h^{\omega}=\left(z^{\omega}, q^{\omega}, \dot{q}^{\omega}\right)$ of $L^{\omega}$ homoclinic to $O_{r}^{\omega}$ with

$$
r \leqslant \frac{1}{\omega} \sqrt{\frac{E_{\infty}}{a}} ;
$$

- The orbits $h^{\omega}$ converge to $M$ in configuration space:

$$
\left\|q^{\omega}\right\|_{\infty} \underset{\omega \rightarrow \infty}{\longrightarrow} 0
$$

- The function $\omega \longrightarrow \int d^{2}\left(z^{\omega}, z_{0}\right)$ is bounded;

- For any sequence $\omega_{n} \longrightarrow \infty$, there is a subsequence $p_{n}$, a finite number $m$ of orbits $Z^{i}$ of $L_{0}$ homoclinic to $z_{0}$ and $m$ sequences $t_{p}^{i}$ such that $\lim _{p \rightarrow \infty}\left(t_{p}^{i+1}-\right.$ $\left.t_{p}^{i}\right)=\infty$ and

$$
z^{\omega_{p}}\left(t-t_{p}^{i}\right) \underset{p \rightarrow \infty}{\stackrel{C_{l o c}^{1}}{\longrightarrow}} Z^{i}(t) .
$$

- If $\omega$ is large enough and fixed, there is an $\epsilon>0$ such that any Lagrangian system $\tilde{L}$ satisfying $\left\|\tilde{L}-L^{\omega}\right\|_{C_{\tilde{h}}^{3}} \leqslant \epsilon$ also has a saddle-center fixed point with a center manifold and an orbit $\tilde{h}$ homoclinic to this center manifold and such that $\tilde{E}(\tilde{h}) \leqslant E_{\infty}$.

\section{Remarks.}

1. The limit configuration space $M=M \times\{0\}$ is not invariant for $L^{\omega}$ hence the fixed point $\left(z_{0}, 0,0\right)$ does not have any homoclinic orbit in general (its stable and unstable manifold have dimension $n$ in a $2 n+1$-dimensional energy shell).

2. The energy $E^{\omega}\left(h^{\omega}\right)$ is bounded, but does not converge to zero, or at least we can not prove that it does. It should be interesting to understand whether this is only a side effect due to our approach, or whether it has a physical meaning. 
3. It should be possible, when $M$ is not simply connected, to prove that the $z^{\omega}$ is actually converging to a single homoclinic of $L_{0}$.

4. The hypothesis HG1 loc is an unpleasant restriction, assumed in order that Theorem 1 can be readily applied. It is not hard to see however that even without this assumption a saddle-center exists in $L^{\omega}$ for large $\omega$, and it may be possible using the techniques of Section 1.1 to prove that the phenomenon described in the application still occurs.

5. Adding more than one degree of freedom makes things much harder. Even in the ideal case where a center manifold foliated by quasi-periodic tori would exist, there would remain the problem that the intersection between the center manifold and an energy shell would contain families of such quasi-periodic tori, in contrast with our situation where each periodic orbit is the intersection between its energy shell and the center manifold. Moreover, this ideal case is not as rigid as our case, since some of the invariant tori are usually destroyed by a perturbation.

6. The classical pendulum is described by the Lagrangian

$$
G_{0}(\theta, \dot{\theta})=\|\dot{\theta}\|^{2}+(1-\cos \theta), \quad \theta \in S^{1}
$$

It is well known that integrability can be destroyed and chaotic behavior turned on by a time-dependent small perturbation. Let us consider a system

$$
L^{\omega}(\theta, \dot{\theta}, q, \dot{q})=\dot{q}^{2}-\omega^{2} q^{2}+G(\theta, \dot{\theta}, q), \quad(\theta, q) \in S^{1} \times \mathbb{R}
$$

with $G(\theta, \dot{\theta}, 0)=G_{0}(\theta, \dot{\theta})$, satisfying the hypotheses of the application. The homoclinic orbit obtained by the application can be made transversal by a small perturbation of $G$. This is a new way, also physically relevant, to introduce chaotic behavior in the classical pendulum.

Proof. We are interested in trajectories located around $q=0$, and it is first necessary to change the Lagrangian function outside a neighborhood of $q=0$. We need a smooth function $\varphi:[0, \infty] \longrightarrow[0,1]$ such that $\left.\varphi\right|_{[0,1]}=1$ and $\left.\varphi\right|_{[2, \infty)}=0$ and $0 \geqslant \varphi^{\prime} \geqslant-2$. Let us fix $\delta>0$ and define

$$
G_{\delta}(z, q)=\varphi(q / \delta) G(z, q)+(1-\varphi(q / \delta)) G_{0}(z)
$$

It is clear that $G_{\delta}$ satisfies HG1 when $\delta$ is small enough. To check the other hypotheses of Theorem 1 let us first notice that hypothesis [HG1 loc] gives a constant $C$ such that

$$
\left|G-G_{0}\right| \leqslant 2 C \delta d^{2}\left(z, z_{0}\right) \quad \text { and } \quad\left|\frac{\partial G}{\partial q}\right| \leqslant C d^{2}\left(z, z_{0}\right)
$$


when $|q| \leqslant 2 \delta$. It follows from the first estimate above that $G_{\delta}(\theta, q) \geqslant b d^{2}\left(z, z_{0}\right) / 2$ when $\delta$ is small enough. In view of the calculation

$$
\begin{aligned}
G_{\delta}-\frac{1}{2} q \frac{\partial G_{\delta}}{\partial q}= & \varphi(q / \delta) G+(1-\varphi(q / \delta)) G_{0} \\
& -\frac{1}{2} q\left(\varphi(q / \delta) \frac{\partial G}{\partial q}+\left(G-G_{0}\right) \varphi^{\prime}(q / \delta) / \delta\right) \\
\geqslant & G_{0}-\left(\varphi(q / \delta)-\varphi^{\prime}(q / \delta)\right)\left|G-G_{0}\right|-\delta \varphi(q / \delta)\left|\frac{\partial G}{\partial q}\right| \\
\geqslant & G_{0}-6 \delta C d^{2}\left(z, z_{0}\right),
\end{aligned}
$$

the hypotheses HG2 and HG3 are both satisfied with the constant $b / 3$ when $\delta$ is small enough. We also obtain that

$$
U_{\delta}=G_{0}(z)-2 C \delta d^{2}\left(z, z_{0}\right) \leqslant G_{\delta} \leqslant G_{0}(z)+2 C \delta d^{2}\left(z, z_{0}\right)=W_{\delta},
$$

and (3) yields

$$
I\left(W_{\delta}\right)-I\left(U_{\delta}\right) \leqslant C \delta,
$$

where $C$ is a new constant that does not depend on $\delta$. We are now in a position to apply Theorem 1 to $L_{\delta}^{\omega}=a\left(v^{2}-\omega^{2} q^{2}\right)+G_{\delta}(z, q)$, and obtain an orbit $h_{\delta}^{\omega}=\left(z_{\delta}^{\omega}, q_{\delta}^{\omega}, \dot{q}_{\delta}^{\omega}\right)$ homoclinic to $O_{r}$ with $r \leqslant B \sqrt{\delta / \omega}$, where $B$ is a constant that depends neither on $\omega$ nor on $\delta$. We shall use energy conservation to localize the obtained homoclinic. Let us consider the function $G_{q}: z \longmapsto G(z, q)$ as a lagrangian on $T M$, and let $E_{q}$ be the associated energy. A simple calculation in local coordinates shows that the energy function $E_{\delta}^{\omega}$ associated with $L_{\delta}^{\omega}$ is

$$
E_{\delta}^{\omega}(z, q)=a\left(v^{2}+\omega^{2} q^{2}\right)+\varphi(q / \delta) E_{q}(z)+(1-\varphi(q / \delta)) E_{0}(z) .
$$

The term $\varphi(q / \delta) E_{q}(z)+(1-\varphi(q / \delta)) E_{0}(z)$ is clearly bounded from below by a constant that does not depend on $\delta \leqslant 1$. Writing energy conservation along $h_{\delta}^{\omega}$ thus yields

$$
\omega^{2}\left|q_{\delta}^{\omega}\right|^{2} \leqslant B^{2} \delta \omega+C .
$$

The homoclinic $h_{\delta}^{\omega}$ is thus an orbit of $L^{\omega}$ if

$$
\omega^{2} \delta^{2} \geqslant B^{2} \delta \omega+C
$$

Let us now choose $\delta=\beta / \omega$, where $\beta$ is a sufficiently large fixed number, the inequality above is satisfied and the homoclinic $h^{\omega}=h_{\beta / \omega}^{\omega}=\left(z^{\omega}, q^{\omega}, \dot{q}^{\omega}\right)$ is a trajectory of $L^{\omega}$, of bounded energy $E^{\omega}\left(h^{\omega}\right) \leqslant E^{\infty}=a B^{2} \beta$, and satisfying

$$
\int_{\mathbb{R}} G_{\beta / \omega}\left(h_{\omega}\right)-\frac{1}{2} q^{\omega} \frac{\partial G_{\beta / \omega}}{\partial q}\left(h^{\omega}\right) \leqslant I\left(W_{\beta / \omega}\right) .
$$

In view of HG3, this estimate yields

$$
\int d^{2}\left(z^{\omega}, z_{0}\right) \leqslant C
$$


The function $G_{q}(z)=G(z, q)$ is a fiberwise convex Lagrangian on $T M$ for all $q$. Let us call $Y_{q}$ the associated vector field. The curves $z^{\omega}$ satisfy the Euler-Lagrange equation

$$
\dot{z}^{\omega}(t)=Y_{q^{\omega}(t)}\left(z^{\omega}(t)\right)
$$

hence $z^{\omega}$ is bounded in $C^{1}(\mathbb{R}, T M)$. Moreover, since $\left\|q^{\omega}\right\| \longrightarrow 0$, any limit curve $z^{\infty}$ of $z^{\omega}$ satisfies the Euler-Lagrange equation

$$
\dot{z}^{\infty}(t)=Y_{0}\left(z^{\infty}(t)\right)
$$

where $Y_{0}$ is the Euler-Lagrange vector field of $G_{0}$. It is not hard to see that there is a constant $C>0$ independent of $\omega$ such that all orbit $Z$ of $G_{0}$ homoclinic to $z_{0}$, satisfies $\int d^{2}\left(Z, z_{0}\right) \geqslant C$, and all orbit $X=(Z, Q, \dot{Q})$ of $L^{\omega}$ homoclinic to the center manifold $z=z_{0}$ and lying in $E^{\omega} \leqslant E^{\infty}$ satisfies $\left\|d\left(Z, z_{0}\right)\right\|_{\infty} \geqslant C$. One now applies the concentration compactness principle, see [29], 4.3, to the function $d^{2}\left(z^{\omega}(t), z_{0}\right)$ in order to prove the last point of the theorem, see [14] for the use of concentration compactness with homoclinic orbits. In our situation, vanishing is impossible since $\left\|d^{2}\left(z^{\omega}, z_{0}\right)\right\|_{\infty} \geqslant C^{2}$, while only a finite number of bumps can appear since each bump satisfies $\int d^{2}\left(Z^{i}, z_{0}\right) \geqslant C$. To finish, the persistence is a direct consequence of Theorem 2 .

\section{Lagrangian and Hamiltonian systems}

We recall in this section some standard facts abound Lagrangian and Hamiltonian systems. This is an opportunity to introduce some notations and to state a simple estimate of the energy function that will be used throughout the paper.

Let $N$ be a manifold, $T N \stackrel{\pi}{\longrightarrow} N$ the tangent bundle and $T^{*} N \stackrel{\pi^{*}}{\longrightarrow} N$ the cotangent bundle. The lifting $\partial x$ of a curve $x: \mathbb{R} \longrightarrow N$ is the curve

$$
\begin{aligned}
\partial x: \mathbb{R} & \longrightarrow T N \\
t & \longmapsto d x(t, 1) .
\end{aligned}
$$

We consider a smooth Lagrangian function $L: T N \longrightarrow \mathbb{R}$, that is convex on each fiber with uniformly positive definte Hessian. The fiber derivative $L_{v}$ of $L$ is well defined, and the application

$$
\begin{aligned}
\phi: T N & \longrightarrow T^{*} N \\
v & \longmapsto L_{v}(v)
\end{aligned}
$$

is a diffeomorphism. We can associate to $L$ an energy function

$$
\begin{aligned}
E: T N & \longrightarrow \mathbb{R} \\
v & \longmapsto\langle\phi(v), v\rangle-L(v)
\end{aligned}
$$

and a Hamiltonian function

$$
\begin{aligned}
H: T^{*} N & \longrightarrow \mathbb{R} \\
p & \longmapsto E \circ \phi^{-1}(p)=\left\langle v, \phi^{-1}(v)\right\rangle-L\left(\phi^{-1}(v)\right) .
\end{aligned}
$$


There is a canonical symplectic structure $\Omega$ on $T^{*} M$, and we associate to $H$ its Hamiltonian vector field $X$ defined by the equation $i_{X} \Omega=-d H$. Let us define the action $\mathcal{L}(x)$ of a smooth curve $x:\left[t_{0}, t_{1}\right] \longrightarrow N$

$$
\mathcal{L}(x)=\int_{t_{0}}^{t_{1}} L(\partial x(t)) d t,
$$

we say that $x$ is a trajectory of $L$ if it is a critical point of the action with respect to fixed endpoints variations. A curve $x: \mathbb{R} \longrightarrow N$ is a trajectory of $L$ if and only if its restrictions to finite time intervals are trajectories of $L$. We will pay special attention to the periodic trajectories of $L$. Let $T>0$ be a fixed period, a $T$-periodic curve $x: \mathbb{R} \longrightarrow N$ is a trajectory of $L$ if and only if the loop $x_{\mid[0, T]}$ is a critical point of

$$
\mathcal{L}_{T}(x)=\int_{0}^{T} L(\partial x)
$$

on $C_{T}^{1}=\left\{x \in C^{1}([0, T], M) / x(0)=x(T)\right\}$. There is a one to one correspondence between trajectories $x$ of $L$ and integral curves $z$ of $X$, given by

$$
x \longrightarrow z=\phi(\partial x), \quad z \longrightarrow x=\pi^{*}(z) .
$$

As a consequence, there is a vector-field $Y$ on $T M$ such that $x$ is a trajectory of $L$ if and only if $\partial x$ is an integral curve of $Y$, and we have

$$
Y(z)=\left(d \phi_{z}\right)^{-1}(X(\phi(z))
$$

In any canonical chart $(q, v)$ of $T M$, the trajectories of $L$ satisfy the Euler-Lagrange equations

$$
\frac{d}{d t} \frac{\partial L}{\partial v}(q(t), \dot{q}(t))=\frac{\partial L}{\partial q}(q(t), \dot{q}(t)) .
$$

The Hamiltonian function $H$ is invariant along integral curves of $X$, and the energy $E$ is invariant along integral curves of $Y$ hence $E(\partial x)$ is constant if $x$ is a trajectory of $L$. This construction can be reversed. Let $H: T^{*} N \longrightarrow \mathbb{R}$ be a Hamiltonian function. If the mapping

$$
\begin{aligned}
\psi: T N & \longrightarrow T N \\
z & \longmapsto H_{v}(z)
\end{aligned}
$$

is a diffeomorphism, which happens when $H$ is fiberwise convex and proper, we define

$$
L(z)=\left(z, \psi^{-1}(z)\right)-H\left(\psi^{-1}(z)\right),
$$

the associated mapping $\phi$ is the diffeomorphism $\phi=\psi^{-1}$, and the correspondence described above between orbits of $L$ and integral curves of $H$ holds. Let us now come back to our main subject of interest and estimate the energy $E$ associated to (1). We assume [HG1-4] and [HL]. Note that the configuration space of $L$ is $N=M \times \mathbb{R}$. 
Lemma 1 Let L be a Lagrangian given by (1), satisfying [HG1-4] and [HL]. There is a constant $C>0$ such that

$$
\left|E(z, q, v)-a\left(v^{2}+\omega^{2} q^{2}\right)\right| \leqslant C d^{2}\left(z, z_{0}\right)
$$

for all $(z, q, v) \in T M \times \mathbb{R} \times \mathbb{R}$.

Proof. Let us consider the energy $E_{\infty}$ on $T M$ associated with the Lagrangian $G_{\infty}$ as defined in [HG4]. It can be computed in local coordinates that

$$
E(z, q, v)=a\left(v^{2}+\omega^{2} q^{2}\right)+E_{\infty}(z)
$$

when $z \notin B$, and that

$$
E_{\infty}(z)=\alpha\|z\|^{2}
$$

outside $K$. Recall that $K$ and $B$ are defined in [HG4]. It follows that the function

$$
\left|E_{\infty}(z)\right| / d^{2}\left(z, z_{0}\right)
$$

is bounded at infinity, and it can be checked from [HG1] (using local expression (10) below) that it is bounded around $z_{0}$, and thus bounded. It follows that the function

$$
\left|E(z, q, v)-a\left(v^{2}+\omega^{2} q^{2}\right)\right| / d^{2}\left(z, z_{0}\right)
$$

is bounded outside $B$. It also follows from [HG1] and the local expression (10) below that this function is bounded in a neighborhood of $B \cap\left\{z=z_{0}\right\}$, and thus bounded everywhere.

\section{Sketch of proof of Theorem 1}

We obtain the homoclinic orbit as limit set of a sequence of periodic orbits obtained by a variational method. We first have to solve a technical difficulty. The statement of Theorem 1 involves no growth conditions while such conditions are needed to define appropriate functionals. These conditions can be artificially obtained by changing the Hamiltonian at infinity, since the behavior we are describing is localized in a compact zone $E \leqslant E_{0}$, where $E$ is the (proper) energy function and

$$
E_{0}=\frac{\omega}{2 \pi}(I(W)-I(U)) .
$$

Proposition 1 If the conclusions of Theorem 1 hold for any Lagrangian function satisfying all the hypotheses of Theorem 1 and the additional hypothesis [HG4], then Theorem 1 holds.

This proposition is proved in Section 4 by changing the Lagrangian function at infinity. It is thus sufficient to prove Theorem 1 for Lagrangian functions satisfying the additional Hypothesis [HG4]. We will use periodic orbits of

$$
L_{l}(z, q, v)=a\left(v^{2}-l^{2} \omega^{2} q^{2}\right)+G(z, q, v), \quad(z, q, v) \in T M \times \mathbb{R} \times \mathbb{R}
$$


obtained as critical points of the Lagrange action functional

$$
\mathcal{L}_{l}(X)=\int_{0}^{T} L_{l}(\partial X)
$$

defined on $T$-periodic loops. The critical points of $\mathcal{L}_{l}$ are precisely the $T$-periodic solutions of $L_{l}$. We will see in Section 8 , that it is possible to find a critical value $c_{T}(l)$ of $\mathcal{L}_{l}$ for all $T=2 \pi \tau / \omega, \tau \in \mathbb{N}$, and all $l \in(1,1+1 / \tau)$. This critical value satisfies

$$
I_{T}(U) \leqslant c_{T}(l) \leqslant I_{T}(W),
$$

the numbers $I_{T}(U)$ are defined in Section 5 together with the numbers $I(U)$. Since the function $l \longrightarrow c_{T}(l)$ is non-increasing, there is an $l(T) \in(1,1+1 / \tau)$ such that $c_{T}^{\prime}(l(T))$ exists and $\left|c_{T}^{\prime}(l(T))\right| \leqslant \tau\left(I_{T}(W)-I_{T}(U)\right)$. We use this to find a critical point $X_{T}=\left(\theta_{T}, q_{T}\right)$ of $\mathcal{L}_{l(T)}$ at level $c_{T}(l(T))$ such that

$2 l(T) a \omega^{2}\left\|q_{T}\right\|_{2}^{2}=\left|\frac{\partial \mathcal{L}_{l}\left(X_{T}\right)}{\partial l}\right|_{l(T)} \leqslant 1+\left|c_{T}^{\prime}(l(T))\right| \leqslant \tau\left(I_{T}(W)-I_{T}(U)\right)+1$.

The periodic orbit we obtain is not trivial i.e. $\theta_{T} \not \equiv \theta_{0}$, because it has nonzero action. All this is detailed in Section 8, where we prove

Proposition 2 For all $\tau \in \mathbb{N}$ and $T=2 \pi \tau / \omega$, there exist a parameter $l(T)$ in the interval $(1,1+1 / \tau)$ and a trajectory $X_{T}=\left(\theta_{T}, q_{T}\right)$ of $L_{l(T)}$ such that

$$
\begin{aligned}
\frac{1}{T}\left\|q_{T}\right\|_{2}^{2} & \leqslant \frac{1}{4 \pi a \omega}\left(I_{T}(W)-I_{T}(U)+\frac{1}{\tau}\right), \\
\mathcal{L}_{l(T)}\left(X_{T}\right) & =c_{T}(l(T)), \\
\theta_{T} & \neq \equiv \theta_{0} .
\end{aligned}
$$

We now use the periodic orbits given by Proposition 2 to build the homoclinic orbit. Since $I(U)=\liminf _{T \rightarrow \infty} I_{T}(U)$, see Section 5, we can extract a subsequence $T_{n}$ of $T$ such that

$$
\frac{1}{T_{n}}\left\|q_{T_{n}}\right\|_{2}^{2} \longrightarrow r^{2} / 2
$$

with a radius

$$
r \leqslant \sqrt{\frac{I(W)-I(U)}{2 \pi a \omega}}
$$

We obtain a sequence $X_{n}$ of $T_{n}$-periodic orbits of $L_{l\left(T_{n}\right)}$ which satisfies

- $l\left(T_{n}\right) \longrightarrow 1$

- $T_{n} \longrightarrow \infty$,

- $\mathcal{L}_{l\left(T_{n}\right)}\left(X_{n}\right) \leqslant I(W)$,

- $\left\|q_{n}\right\|^{2} / T_{n} \longrightarrow r^{2} / 2$,

- $\theta_{n} \not \equiv \theta_{0}$,

and we prove in Section 7 that a homoclinic orbit can be found as an accumulation point of this sequence. Moreover, this homoclinic orbit satisfies all the conclusions of Theorem 1. 


\section{Control at infinity}

All the systems considered in this paper are autonomous, and preserve an energy function. It is thus possible to change the Lagrangian function at infinity without changing the flow on prescribed compact energy shells. This observation is the basis of the proof of Proposition 1. The details are not simple since fiberwise convexity has to be preserved during the process. We now prove Proposition 1.

Let us consider a Lagrangian function $L=a\left(v^{2}-\omega^{2} q^{2}\right)+G$ satisfying all the hypotheses of Theorem 1 . We build a new Lagrangian function that is equal to the old one on $E \leqslant E_{0}$ and that satisfies all the hypothesis of Theorem 1 and [HG4]. Recall that

$$
E_{0}=\frac{\omega}{2 \pi}(I(W)-I(U)) .
$$

First step: Let $K_{0}$ be a compact subset of $T M$, we first build a function $G_{1}$ on $T M \times \mathbb{R}^{2}$ such that

$$
\begin{gathered}
G_{1}(z, q, v)=G(z, q, v) \quad \text { when } z \in K_{0}, \\
G_{1}(z, q, v)=\alpha\|z\|^{2} \quad \text { when } z \notin K
\end{gathered}
$$

for some $\alpha>0$ and some compact set $K \subset T M$, and $G_{1}+a v^{2}$ is fiberwise convex. Let us set $d=\sup _{z \in K_{0}}\|z\|$. There exists a $d_{1}>d$ such that for all $\alpha>0$ there exists a convex function $f_{\alpha}:[0, \infty) \longrightarrow[0, \infty)$ satisfying $f_{\alpha}(x)=0$ when $x \leqslant d$ and $f_{\alpha}(x)=\alpha x^{2}$ when $x \geqslant d_{1}$. We now consider a function $\varphi: \mathbb{R}^{+} \longrightarrow[0,1]$ such that $\varphi(x)=1$ when $x \leqslant d_{1}$ and $\varphi(x)=0$ when $x \geqslant d_{2}$ for some $d_{2}>d_{1}$ and we set

$$
G_{1}(z, q, v)=\varphi(\|z\|) G(z, q, v)+f_{\alpha}\left(\|z\|^{2}\right) .
$$

It is easy to see that the function $G_{1}+a v^{2}$ is fiberwise convex except maybe on the compact set $d_{1} \leqslant\|z\| \leqslant d_{2}$. To prove that $G_{1}+a v^{2}$ is also fiberwise convex on $d_{1} \leqslant\|z\| \leqslant d_{2}$ we will use a local canonical chart $z=(\theta, \nu)$ of $T M$ and prove that the function $G_{1}+a v^{2}$ is convex in $(\nu, v)$ on $d_{1} \leqslant\|z\| \leqslant d_{2}$. We first note that

$$
d_{v}^{2}\left(G_{1}+a v^{2}\right)=\varphi(\|z\|) d_{v}^{2} G+2 a
$$

is positive since $d_{v}^{2} G+2 a$ is positive. On the other hand, it is not hard to see that given $\beta>0$ one can take $\alpha$ large enough so that $d_{\nu}^{2} G_{1} \geqslant \beta I d$ on $d_{1} \leqslant\|z\| \leqslant d_{2}$. Since the cross derivatives $d_{\nu} d_{v}\left(G_{1}+a v^{2}\right)$ do not depend on $\alpha$, one can check that the Hessian

$$
\left[\begin{array}{cc}
d_{\nu}^{2}\left(G_{1}+a v^{2}\right) & d_{v} d_{\nu}\left(G_{1}+a v^{2}\right) \\
d_{\nu} d_{v}\left(G_{1}+a v^{2}\right) & d_{v}^{2}\left(G_{1}+a v^{2}\right)
\end{array}\right]
$$

is positive definite when $\alpha$ is large enough. The function $G_{1}+a v^{2}$ is thus fiberwise convex, as well as the function $L_{1}=a\left(v^{2}-\omega^{2} q^{2}\right)+G_{1}$. The function $G_{1}$ satisfies [HG1-3] with the same constant $b$. Let us define the functions

$$
U_{1}(z)=\varphi(\|z\|) U(z)+f_{\alpha}\left(\|z\|^{2}\right),
$$

and $W_{1}$ in the same way, so that

$$
U_{1} \leqslant G_{1} \leqslant W_{1} .
$$


If $\alpha$ has been chosen large enough, the functions $U_{1}$ and $W_{1}$ are fiberwise convex and satisfy

$$
U_{1}(z)=U(z) \text { and } W_{1}(z)=W(z) \quad \text { when } z \in K_{0}
$$

and

$$
U_{1}(z)=W_{1}(z)=\alpha\|z\|^{2} \quad \text { when } z \notin K .
$$

If $K_{0}$ is sufficiently large, the definition of $I$ is $I(U)=I\left(U_{1}\right)$ and $I(W)=I\left(W_{1}\right)$ (see Definition 2 of Section 5). So the maximal energy $E_{0}$ has not been changed.

Second step: We now want to control the behavior for large $v$. Let $B_{0}$ be a compact subset of $T M \times \mathbb{R}^{2}$, we will define a function $\tilde{G}$ such that $\tilde{G}=G_{1}$ on $B_{0}$ and $\tilde{G}=U_{1}$ outside a compact subset $B \supset B_{0}$. To do this we first observe that $U_{1}-G_{1}$ is bounded, which easily implies that $d_{v} G_{1}$ is bounded since $d_{v}^{2} G_{1} \geqslant-2 a$. We now take a compactly supported function $\psi: \mathbb{R}^{2} \longrightarrow[0,1]$ such that $\psi(q, v)=1$ when there exists a $z \in T M$ with $(z, q, v) \in B_{0}$, and such that $\|d \psi\|_{C^{1}} \leqslant \epsilon$ and $q d_{q} \psi+v d_{v} \psi \leqslant 0$. The function

$$
\tilde{G}(z, q, v)=\psi(q, v) G_{1}(z, q, v)+(1-\psi(q, v)) U_{1}(z),
$$

satisfies [HG4] with $G_{\infty}=U_{1}$. With the notation $z=(\theta, \nu)$, we derive

$$
\begin{aligned}
d_{\nu}^{2} \tilde{G} & =\psi d_{\nu}^{2} G_{1}+(1-\psi) d_{\nu}^{2} U_{1} \\
d_{v} d_{\nu} \tilde{G} & =\psi d_{v} d_{\nu} G_{1}+d_{v} \psi\left(d_{\nu} G_{1}-d_{\nu} U_{1}\right) \\
d_{v}^{2} \tilde{G} & =\psi d_{v}^{2} G_{1}+d_{v}^{2} \psi\left(G_{1}-U_{1}\right)+2 d_{v} \psi d_{v} G_{1},
\end{aligned}
$$

and get

$$
\left\|d_{(v, \nu)}^{2}\left(\tilde{G}+a v^{2}\right)-\psi d_{(v, \nu)}^{2}\left(G_{1}+a v^{2}\right)-(1-\psi) d_{(v, \nu)}^{2}\left(U_{1}+a v^{2}\right)\right\|_{\infty} \underset{\epsilon \rightarrow 0}{\longrightarrow} 0
$$

which implies that $\tilde{G}$ is fiberwise convex when $\epsilon$ is small enough. The function $\tilde{G}$ moreover satisfies [HG1-2] with the same constant $b$ and [HG3] follows from :

$$
\begin{aligned}
\tilde{G}-\frac{1}{2}\left(q d_{q} \tilde{G}+v d_{v} \tilde{G}\right)= & \psi\left(G_{1}-\frac{1}{2}\left(q d_{q} G_{1}+v d_{v} G_{1}\right)\right) \\
& +(1-\psi) U_{1}+\frac{1}{2}\left(U_{1}-G_{1}\right)\left(q d_{q} \psi+v d_{v} \psi\right) \\
& \geqslant \psi b d^{2}\left(z, z_{0}\right)+(1-\psi) b d^{2}\left(z, z_{0}\right)=b d^{2}\left(z, z_{0}\right) .
\end{aligned}
$$

The function $\tilde{L}=a\left(v^{2}-\omega^{2} q^{2}\right)+\tilde{G}$ satisfies all the hypotheses of Theorem 1 with the same constants $a, b$ and $\omega$, and with $U$ and $W$ replaced by $U_{1}$ and $W_{1}$. Let us assume that $K_{0}$ and $B_{0}$ have been taken large enough so that

$$
\left\{E \leqslant E_{0}\right\} \subset B_{0} \cap\left(K_{0} \times \mathbb{R}^{2}\right) .
$$

If the conclusions of Theorem 1 hold for $\tilde{L}$, they give the existence of a homoclinic of energy below $E_{0}$, this orbit is also an orbit of $L$ since the function have not been changed in this region, the conclusion of Theorem 1 thus hold for $L$. 


\section{Systems with a hyperbolic fixed point}

We now define the number $I(U)$ for a fiberwise convex Lagrangian $U: T M \longrightarrow \mathbb{R}$ satisfying [HU1-2]. These hypotheses imply that $z_{0}$ is a hyperbolic fixed point of the system, see Section 6, and that it has a homoclinic orbit. Homoclinics for this kind of Lagrangian were first studied variationally by Bolotin [6], and then by several authors (see e.g. [3] , [27]). These works have also been extended to more general Hamiltonian systems in [17] and [11], [12]. We just give here a presentation of these results that will be useful in the proof of Theorem 1. We shall first study Lagrangians $U$ with the additional hypothesis

HU3 : There exists an $\alpha>0$ such that $U(z)=\alpha\|z\|^{2}$ outside a compact set of $T M$.

Although we are interested mainly in the homoclinic orbit, we shall use a variational setting for $T$-periodic orbits of $U$ : Let $\Lambda_{T}$ be the manifold of $H^{1}$-loops

$$
\gamma: S_{T}=\mathbb{R} / T \mathbb{Z} \rightarrow M,
$$

the action functional

$$
\begin{aligned}
\mathcal{U}_{T}: \Lambda_{T} & \longrightarrow \mathbb{R} \\
\gamma & \longmapsto \int_{0}^{T} U(\partial \gamma(t)) d t
\end{aligned}
$$

is smooth and satisfies the Palais-Smale condition. We note $H$ the rational cohomology. A pointed set $(S, s)$ is a set $S$ with a distinguished element $s \in S$. We will use the notation $H(S, s)$ for the relative cohomology $H(S,\{s\})$. For any closed subset $S$ of $\Lambda_{T}$ containing the constant loop $\theta \equiv \theta_{0}$ we consider the morphism

$$
i_{S}^{*}: H\left(\Lambda_{T}, \theta_{0}\right) \longrightarrow H\left(S, \theta_{0}\right)
$$

associated with the inclusion.

Definition 1 We call $\Sigma_{T}$ the family of all compact subsets $\sigma$ of $\Lambda_{T}$ containing $\theta_{0}$ and having induced cohomology, i.e. such that $i_{\sigma}^{*} \neq 0$.

The distinguished level

$$
I_{T}(U)=\inf _{\sigma \in \Sigma_{T}} \sup _{\sigma} \mathcal{U}_{T}
$$

satisfies:

Lemma 2 There exists a constant $M>0$ independent of $T$ such that $0<I_{T}(U) \leqslant$ $M$.

Proof. To prove that $I_{T}(U)>0$, we take a small disk $D \in M$ centered at $\theta_{0}$, and let $\Lambda_{T}(D)$ be the set of $H^{1}$ loops in $D$. It is not hard to see that $\Lambda_{T}(D)$ is contractible, thus $i_{\Lambda_{T}(D)}^{*}=0$ and $i_{\sigma}^{*}=0$ for all $\sigma \subset \Lambda_{T}(D)$ containing $\theta_{0}$. From this follows that all $\sigma \in \Sigma$ must contain a curve leaving $D$. Such a curve has its action bounded away from 0 . To prove the second inequality, let us introduce the set of loops starting at $\theta_{0}$

$$
\Lambda_{T}^{0}=\left\{\theta(t) \in \Lambda_{T} / \theta(0)=\theta_{0}\right\} .
$$

We need the 
Lemma 3 There exists a compact subset $K \subset \Lambda_{1}^{0}$ such that $i_{K}^{*} \neq 0$.

Proof of Lemma 3. This is very classical, and we shall only outline the proof. If $M$ is not simply connected, there is a non contractible curve $\gamma \in \Lambda_{1}^{0}$. We take $K=\left\{\gamma, \theta_{0}\right\}$, and see that $i_{K}^{*}\left(H^{0}\left(\Lambda_{1}, \theta_{0}\right)\right) \neq 0$. Things are much harder when $M$ is simply connected. Let us set $C=C^{0}\left(S^{1}, M\right)$ and $C^{0}=\{\gamma \in$ $\left.C^{0}\left(S^{1}, M\right) / \gamma(0)=\theta_{0}\right)$, the inclusion $i_{\Lambda_{0}}:\left(\Lambda^{0}, \theta_{0}\right) \longrightarrow\left(\Lambda, \theta_{0}\right)$ is homotopy equivalent to the inclusion $i c:\left(C^{0}, \theta_{0}\right) \longrightarrow\left(C, \theta_{0}\right)$. A theorem of Sullivan gives the existence of infinitely many nonzero rational Betti numbers of the space $C^{0}\left(S^{1}, M\right)$ if $\pi_{1}(M)=0$, see [30], page 46. Then, we consider the Serre fibration

$$
\begin{aligned}
& C \longrightarrow M \\
& \gamma \longmapsto \gamma(0)
\end{aligned}
$$

of fiber $C^{0}$ to prove that $i c^{*}$ is nonzero, hence $i_{\Lambda_{0}}^{*}$ is nonzero. We now use broken geodesics approximation, see [8], to find a compact $K$ representing this cohomology.

Proof of Lemma 2. For any $T \geqslant 1$, we can extend loops in $\Lambda_{1}^{0}$ to $[0, T]$ by fixing them in $\theta_{0}$ outside $[0,1]$, this defines the injection

$$
\begin{aligned}
j_{T}:\left(\Lambda_{1}^{0}, \theta_{0}\right) & \longrightarrow\left(\Lambda_{T}^{0}, \theta_{0}\right) \\
\theta(t) & \longmapsto j_{T}(\theta(t))=\theta(\min (1, t))
\end{aligned}
$$

which is homotopic to the diffeomorphism

$$
\begin{aligned}
\left(\Lambda_{1}^{0}, \theta_{0}\right) & \longrightarrow\left(\Lambda_{T}^{0}, \theta_{0}\right) \\
\theta(t) & \longmapsto S_{T}(\theta(t))=\theta(t / T) .
\end{aligned}
$$

It follows that $j_{T}(K) \in \Sigma_{T}$, thus

$$
I_{T}(U) \leqslant \sup _{j_{T}(K)} \mathcal{U}_{T}=\sup _{K} \mathcal{U}_{1}
$$

because the trajectory $t \longmapsto \theta_{0}$ has zero action. This ends the proof of the lemma since $\sup _{K} \mathcal{U}_{1}$ is a finite number.

There is a $T$-periodic trajectory $\gamma_{T}$ such that $\mathcal{U}_{T}\left(\gamma_{T}\right)=I_{T}(U)$. We shall not prove it since it is very classical, and involves arguments simpler than those of Section 8. Here non-trivial means that $\gamma_{T} \not \equiv \theta_{0}$. We can define the number

$$
I(U)=\liminf _{T \longrightarrow \infty} I_{T}(U) .
$$

There must be a nontrivial homoclinic orbit to $z_{0}$ such that

$$
\int_{-\infty}^{\infty} U(\dot{\gamma}(t)) d t \leqslant I(U),
$$

we obtain it as an accumulation point of the sequence $\gamma_{T}$ of periodic orbits, compare Section 7. The following proposition is useful for applications 
Proposition 3 The function $U \longmapsto I(U)$ is increasing and continuous:

$$
\begin{gathered}
U \leqslant W \Longrightarrow I(U) \leqslant I(W) \\
\left|1-\frac{I(W)}{I(U)}\right| \leqslant \sup _{z} \frac{|W(z)-U(z)|}{b d^{2}\left(z, z_{0}\right)}
\end{gathered}
$$

for all $U$ and $W$ satisfying [HU1-3]. Recall that $b$ is the constant of [HU2].

Proof. The monotonicity is clear, we shall prove regularity. Let us set

$$
\lambda=\sup _{z} \frac{|W(z)-U(z)|}{b d^{2}\left(z, z_{0}\right)},
$$

we obtain using [HU2] that

$$
(1-\lambda) U \leqslant W \leqslant(1+\lambda) U
$$

This yields

$$
(1-\lambda) I_{T}(U) \leqslant I_{T}(W) \leqslant(1+\lambda) I_{T}(U) .
$$

We thus have for any $T$

$$
\left|1-\frac{I_{T}(W)}{I_{T}(U)}\right| \leqslant \lambda,
$$

and we obtain the proposition by taking the limit.

Let us come back to Lagrangian systems $U$ on $T M$ satisfying only [HU1,2] but not [HU3]. The energy function $E_{U}$ is proper and the sets $E_{U}^{e}=\left\{E_{U} \leqslant e\right\}$ are compact. Let $\mathcal{E}_{U}$ be the set of all Lagrangians $U_{1}$ satisfying [HU1-3] and such that $U_{1}=U$ on $E_{U}^{e}$ for some $e>0$. Elements of $\mathcal{E}_{U}$ can be constructed by the methods of Section 4.

Definition 2 For all Lagrangian function $U$ satisfying [HU1,2], we set

$$
I(U)=I\left(U_{1}\right)
$$

for any $U_{1} \in \mathcal{E}_{U}$. The proposition 3 holds for $U$ and $W$ satisfying [HU1,2] with this extended definition of $I$.

Proof. One has to prove that the number $I\left(U_{1}\right)$ does not depend on the choice of the Lagrangian $U_{1} \in \mathcal{E}_{U}$. Let us take two Lagrangians $U_{1}$ and $U_{0}$ in $\mathcal{E}_{U}$, define $U_{t}=t U_{1}+(1-t) U_{0}, t \in[0,1]$, and let $E_{t}$ be the energy function associated with $U_{t}$. There is an energy $e>0$ such that $U_{t}(z)=U(z)$ for all $z \in E_{U}^{e}$ and all $t \in[0,1]$. The Lagrangians $U_{t}$ satisfy [HU1,2] with the same constant $b$, and $U_{t}=\alpha_{t}\|z\|^{2}$ at infinity hence $E_{t}=\alpha_{t}\|z\|^{2}$ at infinity. Since $\alpha_{t}, t \in[0,1]$ is bounded there is a constant $C>0$ independent of $t$ such that $E_{t} \leqslant C d^{2}\left(z, z_{0}\right)$, see Lemma 1. For all $T>0$ there is a $T$-periodic trajectory $\gamma_{T}^{t}$ of $U_{t}$ such that $\mathcal{U}_{T}^{t}\left(\gamma_{T}^{t}\right)=I_{T}\left(U_{t}\right)$. One can build by the methods of Section 4 a Lagrangian $U_{2} \in \mathcal{E}_{U}$ such that $U_{2} \geqslant \max \left(U_{0}, U_{1}\right)$ and thus $U_{2} \geqslant U_{t}$ for all $t \in[0,1]$. It follows that $\mathcal{U}_{T}^{t}\left(\gamma_{T}^{t}\right)=I_{T}\left(U_{t}\right) \leqslant I_{T}\left(U_{2}\right)$ is bounded, and

$$
E_{t}\left(\gamma_{T}^{t}\right) \leqslant \frac{C}{T} \int d^{2}\left(\partial \gamma_{T}^{t}, z_{0}\right) \leqslant \frac{C}{T b} \int U_{t}\left(\partial \gamma_{T}^{t}\right) \leqslant \frac{C^{\prime}}{T} .
$$


As a consequence, there exists a $T_{0}>0$ such that all the periodic orbits $\partial \gamma_{T}^{t}$ with $T \geqslant T_{0}$ are contained in $\left\{E_{t} \leqslant e\right\}$, which is nothing but $E_{U}^{e}$. The curves $\gamma_{T}^{t}$ with $T \geqslant T_{0}$ are thus all trajectories of $U$ and of $U_{0}$, and the value $I_{T}\left(U_{t}\right)$ is critical for the Lagrange action $\mathcal{U}_{T}^{0}$ associated with $U_{0}$. The set of critical values of $\mathcal{U}_{T}^{0}$ has measure zero. This is a non-trivial application of Sard's Theorem, see for example [12], Lemma 3.1, for a result of this kind. On the other hand, we see from Proposition 3 that the function $t \longmapsto I_{T}\left(U_{t}\right)$ is continuous, hence constant since it takes values in a set of measure zero. We have proved that $I_{T}\left(U_{1}\right)=I_{T}\left(U_{0}\right)$ when $T$ is large enough, hence $I\left(U_{1}\right)=I\left(U_{0}\right)$, and the definition is meaningful. We now prove that Proposition 3 holds with this extended definition. Let us consider two Lagrangian functions $U$ and $W$ satisfying [HU1-2]. We use the construction of Section 4 to build distinguished elements of $\mathcal{E}_{U}$ and $\mathcal{E}_{W}$. We take $K_{0}$ containing $E_{U}^{0}$ and $E_{W}^{0}$ in its interior, and define $U_{1} \in \mathcal{E}_{U}$ and $W_{1} \in \mathcal{E}_{W}$ by the same expression (9). It is clear that $U_{1} \leqslant W_{1}$ if $U \leqslant W$, and that $\left|I\left(U_{1}\right)-I\left(W_{1}\right)\right| \leqslant|I(U)-I(W)|$. Proposition 3 for $U_{1}$ and $W_{1}$ thus implies Proposition 3 for $U$ and $W$.

Let us now come back to the full system.

\section{Local structure}

In this section, we focus on the vicinity of the center manifold $z=z_{0}$. Let us define the balls $D_{\delta}=B\left(\theta_{0}, \delta\right) \in M$ and $B_{\delta}=B\left(z_{0}, \delta\right) \in T M$. We will work in a local chart of $M$ around $\theta_{0}$, that is we identify $D_{\delta}$ with a neighborhood of 0 in $\mathbb{R}^{n}$. The local form of the Lagrangian function is

$L(\theta, \nu, q, v)=a\left(v^{2}-\omega^{2} q^{2}\right)+G(\theta, \nu, q, v), \quad(\theta, \nu, q, v) \in D_{\delta} \times \mathbb{R}^{n} \times \mathbb{R} \times \mathbb{R}$,

and we can compute the associated energy function, see Section 2

$$
E(\theta, \nu, q, v)=a\left(v^{2}+\omega^{2} q^{2}\right)+\left(\nu, \frac{\partial G}{\partial \nu}\right)+\left(v, \frac{\partial G}{\partial v}\right)-G
$$

The hypothesis [HG2] implies

[HG2 loc] :

$$
\frac{\partial^{2} G}{\partial(\theta, \nu)^{2}}(0,0, q, v) \geqslant b .
$$

We will only use this local minimizing property in this section. The following lemma will not be used in the sequel, but Lemma 5 below is the key to nontriviality.

Lemma 4 (Description of the local orbit structure) If the hypotheses [HG1] and [HG2 loc] are satisfied, the flow has a saddle-center fixed point $(0,0) \in$ $B_{\delta} \times \mathbb{R}^{2}$ with a 2-dimensional elliptic space and a $2 n$-dimensional hyperbolic space. The center manifold of this saddle-center fixed point is the invariant plane $\{0\} \times \mathbb{R}^{2} \subset B_{\delta} \times \mathbb{R}^{2}$. The flow on the center manifold is linear elliptic, and the center manifold is foliated by the trajectories

$$
O_{r}(t)=(0, r \cos (\omega t),-\omega r \sin (\omega t)) .
$$

Each of these periodic orbits is the intersection between its energy shell and the center manifold, and is hyperbolic with respect to its energy shell (but not with respect to the full phase space). 
Proof. Let $\phi: T M \times \mathbb{R}^{2} \longrightarrow T^{*} M \times \mathbb{R}^{2}$ be the diffeomorphism defined by fiberwise derivation, see Section 2. We have the expression in local coordinates

$$
\phi(\theta, \nu, q, v)=\left(\theta, \frac{\partial G}{\partial \nu}, q, 2 a v+\frac{\partial G}{\partial v}\right),
$$

hence $\phi\left(\{0\} \times \mathbb{R}^{2}\right)=\{0\} \times \mathbb{R}^{2}$ and the Hamiltonian $H=E \circ \phi^{-1}$ can be written

$$
H(\theta, \zeta, q, p)=\frac{1}{4 a} p^{2}+a \omega^{2} q^{2}+R(\theta, \zeta, q, p)
$$

where $R=O\left(\|\theta\|^{2}+\|\zeta\|^{2}\right)$. It follows that the plane $\{0\} \times \mathbb{R}^{2}$ is invariant for the Hamiltonian flow, and foliated by the periodic orbits

$$
\tilde{O}_{r}(t)=(0,0, r \cos (\omega t),-2 a \omega r \sin (\omega t)),
$$

we apply $\phi^{-1}$ to obtain the expression of the associated orbits of $Y$. We now prove hyperbolicity. The hypersurface

$$
\Sigma=\left\{(z, q, v) \in T M \times \mathbb{R}^{2} / q>0 \text { and } v=0\right\}=T M \times \mathbb{R}_{*}^{+}
$$

is transversal to the flow around $\{0\} \times \mathbb{R}_{*}^{+}$, and we define the associated Poincaré return map $\Phi$. Let us fix a $r>0$, we want to study the eigenvalues of modulus 1 of the linearized map $d \Phi(0, r)$. Note that $\Phi_{\mid\{0\} \times \mathbb{R}_{*}^{+}}=I d$, thus $d \Phi(0, r)_{\mid\{0\} \times \mathbb{R}}=I d$. It follows that for all $\epsilon>0$ there is a fully resonant approximation $\Psi$ of $d \Phi(0, r)$ such that

$$
\|\Psi(q, z)-d \Phi(0, r)(q, z)\| \leqslant \epsilon\|z\| .
$$

By fully resonant, we mean that all the eigenvalues of modulus 1 of $\Psi$ are roots of the unity. We can moreover take $\epsilon$ small enough so that $\Psi$ and $d \Phi(0, r)$ have the same number of eigenvalues of modulus 1 . Since $\Psi_{\mid\{0\} \times \mathbb{R}_{*}^{+}}=I d$ there exists a neighborhood of $(0, r) \in \Sigma$ where

$$
|\Phi(z, q)-d \Phi(0, r)(z, q-r)-(0, r)| \leqslant \epsilon\|z\|^{2} .
$$

As a consequence, there exists a function $G_{1}$ satisfying [HG1] and [HG2] with a smaller constant $b_{1}$ and such that Poincaré map $\Phi_{1}$ of the flow associated to

$$
L_{1}(\theta, \nu, q, v)=a\left(v^{2}-\omega^{2} q^{2}\right)+G_{1}(\theta, \nu, q, v)
$$

satisfies $\Phi_{1}(z, q)=(0, r)+\Psi(z, q-r)$ in a neighborhood of $(0, r)$. Let us consider an eigenspace of $\Psi$ associated with a pair of eigenvalues of modulus one, which are therefore root of the unity. This eigenspace is filled with periodic points, moreover given $\delta>0$ there exists a neighborhood of 0 in the eigenspace such that all the points in this neighborhood have their $\Psi$-orbit contained in the zone where $\Phi_{1}(z, q)=(0, r)+\Psi(z, q-r)$, and such that the periodic orbits of $L_{1}$ associated with these $\Phi_{1}$-orbits are contained in $B_{\delta} \times \mathbb{R}^{2}$. We now apply the lemma 5 below to $L_{1}$ and obtain that the periodic orbits we just constructed must be the trivial ones, corresponding to the fixed space $\{0\} \times \mathbb{R}$ of $\Psi$. As a consequence, the linearized Poincaré map $d \Phi(0, r)$ can have no eigenvalue of modulus 1 except the one associated with this fixed space. 
Lemma 5 Let L be the Lagrangian function (1) with a function G satisfying [HG12], there is a two-parameters family of periodic orbits of $L$

$$
O(t)=\left(\theta_{0}, r \cos (\omega t+\phi)\right)
$$

and there exists a $\delta>0$ such that they are the only periodic orbits satisfying $\partial x \in B_{\delta} \times \mathbb{R}^{2}$.

Proof. We work in local coordinates as described above. The trajectories lying in $D_{\delta} \times \mathbb{R}$ satisfy the standard Euler-Lagrange equation

$$
\frac{d}{d t} \frac{\partial G}{\partial \nu}=\frac{\partial G}{\partial \theta}
$$

As a consequence of [HG2 loc] there is a $\delta>0$ such that

$$
\left\langle\nu, \frac{\partial G}{\partial \nu}\right\rangle \geqslant \frac{b}{2}\|\nu\|^{2} \text { and }\left\langle\theta, \frac{\partial G}{\partial \theta}\right\rangle \geqslant \frac{b}{2}\|\theta\|^{2}
$$

in $B_{\delta} \times \mathbb{R}^{2}$. Let us now consider a closed trajectory $(\theta(t), q(t))$ such that $(\theta, \dot{\theta}) \in B_{\delta}$, the equation

$$
\int\left\langle\theta, \frac{\partial G}{\partial \theta}\right\rangle=\int\left\langle\theta, \frac{d}{d t} \frac{\partial G}{\partial \nu}\right\rangle=-\int\left\langle\dot{\theta}, \frac{\partial G}{\partial \nu}\right\rangle
$$

yields

$$
\frac{b}{2} \int\|\theta\|^{2} \leqslant \int\left\langle\theta, \frac{d}{d t} \frac{\partial G}{\partial \nu}\right\rangle \leqslant-\frac{b}{2} \int\|\dot{\theta}\|^{2} .
$$

It follows that $\|\theta\| \equiv\|\dot{\theta}\| \equiv 0$.

\section{Convergence of sequences of periodic orbits}

In this section, we prove the convergence of good sequences of periodic orbits to homoclinic orbits. We first state the strong minimizing property of the subspace $z=z_{0}$.

Lemma 6 Any $T$-periodic trajectory $X=(\theta, q)$ of $L$ satisfies

$$
\mathcal{L}_{T}(X) \geqslant b \int_{0}^{T} d\left(\partial \theta, z_{0}\right)^{2} .
$$

Proof. If $X=(\theta, q)$ is a trajectory, $q$ must satisfy the first Euler-Lagrange equation

$$
2 a\left(\ddot{q}+\omega^{2} q\right)=\frac{\partial G}{\partial q}-\frac{d}{d t}\left(\frac{\partial G}{\partial v}\right) .
$$


If $X$ is closed, we can integrate by parts to write its action

$$
\begin{aligned}
\mathcal{L}(X) & =\int-a q\left(\ddot{q}+\omega^{2} q\right)+G(\partial \theta, q, \dot{q}) \\
& =\int\left(G-\frac{1}{2} q \frac{\partial G}{\partial q}\right)+\int \frac{1}{2} q \frac{d}{d t}\left(\frac{\partial G}{\partial v}\right),
\end{aligned}
$$

and integrating by part again the last term,

$$
\mathcal{L}(X)=\int G-\frac{1}{2} q \frac{\partial G}{\partial q}-\frac{1}{2} \dot{q} \frac{\partial G}{\partial v} \geqslant b \int d\left(\partial \theta, z_{0}\right)^{2} .
$$

This lemma roughly implies that if there exists a sequence of periodic orbits of $L$ of unbounded period and bounded action, there must be an orbit homoclinic to the center manifold. Unfortunately, there is no confinement in the $q$ direction, and we must have some estimate of the $q$ part of the periodic orbits in order to be able to prove convergence. As explained in the sketch of proof, we must allow the parameter $\omega$ to vary. Consider now a sequence $\omega_{n}$ of pulsations, with a limit $\omega$, and the associated Lagrangian and action $L_{n}$ and $\mathcal{L}_{n}$. We have the following convergence property:

Proposition 4 If there exist a constant $M$, a radius $r$ and a sequence $X_{n}=\left(\theta_{n}, q_{n}\right)$ of $T_{n}$-periodic orbits of $L_{n}$ such that

$-T_{n} \longrightarrow \infty$,

- $\mathcal{L}_{n}\left(X_{n}\right) \leqslant M$,

- $\left\|q_{n}\right\|^{2} / T_{n} \longrightarrow r^{2} / 2$,

- $\theta_{n} \not \equiv \theta_{0}$,

then there exists an orbit $X_{\infty}=\left(\theta_{\infty}, q_{\infty}\right)$ homoclinic to $O_{r}$ and such that

$$
\int_{\mathbb{R}} G\left(\partial X_{\infty}\right)-\frac{1}{2} q_{\infty} \frac{\partial G}{\partial q}\left(\partial X_{\infty}\right)-\frac{1}{2} \dot{q}_{\infty} \frac{\partial G}{\partial v}\left(\partial X_{\infty}\right) \leqslant M .
$$

Proof. Since $\theta_{n} \not \equiv \theta_{0}$, Lemma 5 implies that $\partial \theta_{n}$ does not stay in $B_{\delta}$. We can consider $\theta_{n}$ as a periodic curve defined on $\mathbb{R}$, and by changing time origin, we can require that

$$
d\left(\partial \theta_{n}(0), z_{0}\right) \geqslant \delta .
$$

Since the sequence $\mathcal{L}_{n}\left(X_{n}\right)$ is bounded, we obtain from Lemma 6 that the sequence

$$
\int_{-T_{n} / 2}^{T_{n} / 2} d^{2}\left(\partial \theta_{n}, z_{0}\right)
$$

is bounded. Associated with Lemma 1 this yields

$$
E_{n}\left(X_{n}\right)-\frac{a}{T_{n}} \int \dot{q}_{n}^{2}+\omega_{n}^{2} q^{2} \longrightarrow 0 .
$$


On the other side, we obtain using the Euler-Lagrange equations and two integrations by parts that

$$
\frac{a}{T_{n}} \int \dot{q}_{n}^{2}-\omega_{n}^{2} q^{2}=\frac{1}{2 T_{n}} \int q_{n} \frac{\partial G}{\partial q}\left(X_{n}\right)+\dot{q}_{n} \frac{\partial G}{\partial v}\left(X_{n}\right) \longrightarrow 0
$$

because [HG1] and [HG4] imply

$$
q \frac{\partial G}{\partial q}(z, q, v)+v \frac{\partial G}{\partial v}(z, q, v) \leqslant C d^{2}\left(z, z_{0}\right)
$$

Combining these equations and the third hypothesis yields

$$
E_{\infty}=\lim E_{n}\left(X_{n}\right)=2 \lim \frac{a}{T_{n}}\left\|\dot{q}_{n}\right\|_{2}^{2}=2 \lim \frac{a \omega_{n}^{2}}{T_{n}}\left\|q_{n}\right\|_{2}^{2}=a \omega^{2} r^{2}
$$

Since $E_{n}\left(X_{n}\right)$ is a bounded sequence and since $\partial X_{n}$ is an integral curve of $Y_{n}$ the sequence $\partial X_{n}$ is $C^{1}$ - bounded, and by Ascoli's Theorem it has a subsequence converging uniformly on compact sets to a limit $\tilde{X}_{\infty}$ that is an integral curve of $Y$ and thus the lifting of a $L$-trajectory $X_{\infty}$ of energy $E_{\infty}$. Recall that

$$
\int_{-T_{n} / 2}^{T_{n} / 2} d^{2}\left(\partial \theta_{n}, z_{0}\right)
$$

is bounded. It follows that

$$
\int_{-\infty}^{\infty} d^{2}\left(\partial \theta_{\infty}, z_{0}\right)
$$

is finite. Since the curve $\partial \theta_{\infty}$ has bounded derivative this yields

$$
\lim _{t \rightarrow \pm \infty} \partial \theta(t)=z_{0}
$$

Using once more the lemma 1 we get that

$$
a\left(\dot{q}_{\infty}^{2}+\omega^{2} q_{\infty}^{2}\right) \underset{t \rightarrow \pm \infty}{\longrightarrow} E_{\infty}=a \omega^{2} r^{2}
$$

This is the definition we have taken for a homoclinic orbit. The last inequality follows from

$$
\int_{-T_{n} / 2}^{T_{n} / 2} G\left(\partial X_{n}\right)-\frac{1}{2} q_{n} \frac{\partial G}{\partial q}\left(\partial X_{n}\right)-\frac{1}{2} \dot{q}_{n} \frac{\partial G}{\partial v}\left(\partial X_{n}\right)=\mathcal{L}\left(X_{n}\right) \leqslant M
$$

since the integrand is non-negative. 


\section{Existence of periodic orbits}

Let us fix a period $T=2 \pi \tau / \omega, \tau \in \mathbb{N}$. For any $l \in \mathbb{R}$, the functional

$$
\begin{aligned}
\mathcal{Q}_{l}: C_{T}^{\infty}(\mathbb{R}) & \longrightarrow \mathbb{R} \\
x(t) & \longmapsto a \int_{0}^{T} \dot{x}(t)^{2}-l^{2} \omega^{2} x(t)^{2}
\end{aligned}
$$

can be computed using Fourier expansion:

$$
\mathcal{Q}_{l}\left(\sum_{k} q_{k} e^{i k \omega t / \tau}\right)=a T \sum_{k}\left(\frac{k^{2} \omega^{2}}{\tau^{2}}-l^{2} \omega^{2}\right)\left|q_{k}\right|^{2} .
$$

It follows that $\mathcal{Q}_{l}$ can be extended to

$$
E_{T}=H^{1}\left(S_{T}=\mathbb{R} / T \mathbb{Z}, \mathbb{R}\right)
$$

as a continuous quadratic form. It has a two dimensional kernel when $l \in \mathbb{Z} / \tau$, and is non-degenerate for other values of $l$. Let us set

$$
\begin{aligned}
& E^{+}=\left\{q \text { such that } q_{k}=0 \text { when }|k| \leqslant \tau\right\} \\
& E^{-}=\left\{q \text { such that } q_{k}=0 \text { when }|k|>\tau\right\},
\end{aligned}
$$

there is an orthogonal splitting

$$
E_{T}=E^{+} \oplus E^{-},
$$

such that $\pm\left.\mathcal{Q}_{l}\right|_{E^{ \pm}}$is positive definite for all $l \in(1,1+1 / \tau)$. Notice that $E^{-}$is finite dimensional, which is the usual feature of Lagrangian formulations. Recalling that $\Lambda_{T}$ is the manifold of $T$-periodic $H^{1}$ loops, let us define the functionals

$$
\begin{aligned}
\mathcal{G}: A_{T} & =\Lambda_{T} \times E_{T} \\
x(t) & \longrightarrow(\theta(t), q(t)) \longmapsto \int_{0}^{T} G(\partial \theta(t), q(t), \dot{q}(t)) d t .
\end{aligned}
$$

and

$$
\begin{aligned}
\mathcal{L}_{l}: A_{T} & =\Lambda_{T} \times E_{T} \\
x(t)=(\theta(t), q(t)) & \longmapsto \mathbb{R} \\
& \longmapsto \mathcal{L}_{l}(x)=\mathcal{Q}_{l}(q)+\mathcal{G}(x) .
\end{aligned}
$$

We also define the projection $P_{\Lambda}: \Lambda_{T} \times E_{T} \longrightarrow \Lambda_{T}$.

Lemma 7 For any $l$ in the interval $(1,1 / \tau)$, the functional $\mathcal{L}_{l}$ is $C^{1}$ and satisfies the Palais-Smale condition. The critical points of $\mathcal{L}_{l}$ are the T-periodic smooth trajectories of the Lagrangian

$$
L_{l}(z, q, v)=a\left(v^{2}-l^{2} \omega^{2} q^{2}\right)+G(z, q, v), \quad(z, q, v) \in T M \times \mathbb{R} \times \mathbb{R} .
$$


Proof. We will often omit the subscript $l$ in the following proof. Recall that $\Lambda_{T}$ is a smooth manifold, and that the mappings

$$
\begin{aligned}
\exp _{c}: H^{1}\left(\mathcal{O}_{c}\right) & \longrightarrow H^{1}\left(S_{T}, M\right) \\
\xi(t) & \longmapsto \exp (\xi(t))
\end{aligned}
$$

are charts of this manifold, where $c \in C^{\infty}\left(S_{T}, M\right), \mathcal{O}_{c}$ is a sufficiently small neighborhood of the zero section in the bundle $c^{*} T M$ of tangents vectors of $M$ along $c$, and $\exp : T M \longrightarrow M$ is the exponential map associated with some spray on $M$, see [22]. Let $\mu_{T}: \mathbb{R} \longrightarrow S_{T}$ be the natural projection, the induced vector bundle $\mu^{*} c^{*} T M$ is trivial since it is a vector bundle over $\mathbb{R}$, we have the commutative diagram

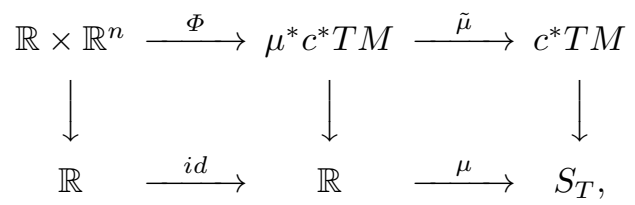

where $\Phi$ is a vector bundle isomorphism and we define the covering

$$
r_{c}=\tilde{\mu} \circ \Phi: \mathbb{R} \times \mathbb{R}^{n} \longrightarrow c^{*} T M .
$$

A $H^{1}$ section $\xi: S_{T} \longrightarrow c^{*} T M$ has a unique lifting $\tilde{\xi}: \mathbb{R} \longrightarrow \mathbb{R}^{n}$ such that the diagram



commutes. Let us take a compact neighborhood $U_{c}$ of the origin in $\mathbb{R}^{n}$ such that $\mathbb{R} \times U_{c} \subset r_{c}^{-1}\left(\mathcal{O}_{c}\right)$, and suppose without loss of generality that $\mathcal{O}_{c}=r_{c}\left(\mathbb{R} \times U_{c}\right)$. The mapping

$$
\begin{aligned}
\rho: H^{1}\left(c^{*} T M\right) & \longrightarrow H^{1}\left([0, T], \mathbb{R}^{n}\right) \\
\xi & \longmapsto \tilde{\xi}_{\mid[0, T]}
\end{aligned}
$$

is a linear isomorphism onto its image $T \tilde{H} \subset H^{1}\left([0, T], \mathbb{R}^{n}\right)$. We will also note $\rho$ the mapping $\left(\rho, i d_{E_{T}}\right)$, and we call $\tilde{H}$ the set $\tilde{H}=T \tilde{H} \cap H^{1}\left([0, T], U_{c}\right)$. Let us define the smooth map

$$
\begin{array}{rl}
\tilde{L}_{c}: \mathbb{R} \times U_{c} \times \mathbb{R}^{n} \times \mathbb{R} \times \mathbb{R} \longrightarrow \mathbb{R} & \mathbb{R}\left(\exp \circ r_{c}(t, \tilde{\xi}),\right. \\
(t, \tilde{\xi}, \nu, q, v) \longmapsto & \left.\quad d_{1}\left(\exp \circ r_{c}\right)(t, \tilde{\xi}) \cdot 1+d_{2}\left(\exp \circ r_{c}\right)(t, \tilde{\xi}) . \nu, q, v\right),
\end{array}
$$

and the functional

$$
\begin{aligned}
\tilde{\mathcal{L}}: H^{1}\left([0, T], U_{c}\right) \times E_{T} & \longrightarrow \mathbb{R} \\
(\tilde{\xi}(t), q(t)) & \longmapsto \int_{0}^{T} \tilde{L}_{c}\left(t, \tilde{\xi}(t), \tilde{\xi}^{\prime}(t), q(t), \dot{q}(t)\right) d t,
\end{aligned}
$$


we have $\mathcal{L}=\tilde{\mathcal{L}} \circ \rho$. One can check from [HG4] and the expression of $\tilde{L}_{c}$ above that the estimates

$$
\begin{array}{r}
\left|\tilde{L}_{c}(t, \tilde{\xi}, \nu, q, v)\right| \leqslant C\left(1+q^{2}+|\nu|^{2}+v^{2}\right) \\
\left|\left(\frac{\partial \tilde{L}_{c}}{\partial \tilde{\xi}}, \frac{\partial \tilde{L}_{c}}{\partial q}\right)(t, \tilde{\xi}, \nu, q, v)\right| \leqslant C\left(1+q+|\nu|^{2}+v^{2}\right) \\
\left|\left(\frac{\partial \tilde{L}_{c}}{\partial \nu}, \frac{\partial \tilde{L}_{c}}{\partial v}\right)(t, \tilde{\xi}, \nu, q, v)\right| \leqslant C\left(1+q^{2}+|\nu|+v\right)
\end{array}
$$

hold on $\mathbb{R} \times U_{c} \times \mathbb{R}^{n} \times \mathbb{R} \times \mathbb{R}$. These growth conditions imply by well-known results (see [24]) that $\tilde{\mathcal{L}}$, and thus $\mathcal{L}$, are continuously differentiable. We also have the local expression of the differential:

$$
d \tilde{\mathcal{L}}(\tilde{\xi}, q)=\int_{0}^{T} \frac{\partial \tilde{L}_{c}}{\partial \tilde{\xi}} d \tilde{\xi}+\frac{\partial \tilde{L}_{c}}{\partial \nu}(d \tilde{\xi})^{\prime}+\frac{\partial \tilde{L}_{c}}{\partial q} d q+\frac{\partial \tilde{L}_{c}}{\partial v}(d q)^{\prime}
$$

and $d \mathcal{L}(\xi, q)=d \tilde{\mathcal{L}}(\tilde{\xi}, q) \circ \rho$. Let us now prove that the Palais-Smale condition is satisfied. We take a Palais-Smale sequence $\left(\theta_{n}, q_{n}\right)$. The sequence

$$
\mathcal{L}_{l}\left(\theta_{n}, q_{n}\right)=\mathcal{Q}_{l}\left(q_{n}\right)+\mathcal{G}\left(\theta_{n}, q_{n}\right)
$$

is bounded. Since $\mathcal{Q}_{l}$ is a non-degenerate quadratic form, there exists an operator $A_{l}: E_{T} \longrightarrow E_{T}$ such that

$$
d \mathcal{Q}_{l}(q) \cdot A_{l} q=\left|\mathcal{Q}_{l}(q)\right| \geqslant C\|q\|_{H^{1}}^{2} .
$$

Let us now write using [HG4] and that $\left\|d \mathcal{L}\left(\theta_{n}, q_{n}\right)\right\|=\epsilon_{n} \longrightarrow 0$

$$
\begin{aligned}
\epsilon_{n}\left\|q_{n}\right\|_{H^{1}} \geqslant d \mathcal{L}_{l}\left(\theta_{n}, q_{n}\right)\left(0, A_{l} q_{n}\right) & =d \mathcal{Q}_{l}\left(q_{n}\right) \cdot A_{l} q_{n}+d \mathcal{G}\left(\xi_{n}, q_{n}\right) \cdot\left(0, A_{l} q_{n}\right) \\
& \geqslant C\left\|q_{n}\right\|_{H^{1}}^{2}+\int \frac{\partial G}{\partial q} \cdot A_{l} q_{n}+\frac{\partial G}{\partial v} \cdot A_{l} \dot{q}_{n} \\
& \geqslant C\left\|q_{n}\right\|_{H^{1}}^{2}-C^{\prime}\left\|q_{n}\right\|_{W^{1,1}} \\
& \geqslant C\left\|q_{n}\right\|_{H^{1}}^{2}-C^{\prime \prime}\left\|q_{n}\right\|_{H^{1}} .
\end{aligned}
$$

It follows that the sequence $\left\|q_{n}\right\|_{H^{1}}$ is bounded. Plugging this into the action

$$
C \geqslant \mathcal{L}_{l}\left(\theta_{n}, q_{n}\right) \geqslant \int G+\mathcal{Q}_{l}\left(q_{n}\right) \geqslant b \int d^{2}\left(\partial \theta_{n}, z_{0}\right)-C\left\|q_{n}\right\|_{H^{1}}^{2}
$$

yields that $\int\left\|\partial \theta_{n}\right\|^{2}$ is also bounded. By a standard application of the theorem of Ascoli, see [22], Lemma 1.4.4, we can find a $C^{0}$-convergent subsequence of $\theta_{n}$, and by extracting another subsequence we can obtain that $q_{n}$ also has a uniform limit. From now on, we will suppose that

$$
\left(\theta_{n}, q_{n}\right) \stackrel{C^{0}}{\longrightarrow}(\theta, q) .
$$

It remains to prove that the limit holds in $\Lambda \times E_{T}$, that is in $H^{1}$-norms. Since the continuous limit $\theta$ can be approximated by a smooth curve $c$, all the curves $\theta_{n}$ lie 
in a single chart $\exp _{c}$ of $\Lambda$ for $n$ large enough. We call $\xi_{n}$ the local representatives of $\theta_{n}$, and we can use the local expressions given above. It is useful to define the mapping

$$
\begin{array}{r}
\Omega_{c}: \mathbb{R} \times U_{c} \times \mathbb{R}^{n} \times \mathbb{R} \times \mathbb{R} \longrightarrow \mathbb{R} \times U_{c} \times \mathbb{R}^{n} \times \mathbb{R} \times \mathbb{R} \\
(t, \tilde{\xi}, \nu, q, v) \longmapsto\left(t, \tilde{\xi}, \frac{\partial \tilde{L}_{c}}{\partial \nu}, q, \frac{\partial \tilde{L}_{c}}{\partial v}\right) .
\end{array}
$$

It is straightforward from the explicit expression of $\tilde{L}_{c}$ and from [HL] that $\Omega_{c}$ is a diffeomorphism, and the estimate

$$
\frac{1}{C}|X|-C \leqslant \Omega_{c}(t, X) \leqslant C(|X|+1)
$$

is a consequence of [HG4]. A theorem of Krasnoselskii implies that the mapping

$$
\begin{aligned}
\Upsilon_{c}: L^{2}\left([0, T], \mathbb{R}^{2 n+2}\right) & \longrightarrow L^{2}\left([0, T], \mathbb{R}^{2 n+2}\right) \\
X(t) & \longmapsto \Omega_{c}(t, X(t))
\end{aligned}
$$

is a homeomorphism. It is not hard to see that the sequence

$$
\left(\frac{\partial \tilde{L}_{c}}{\partial \tilde{\xi}}\left(\tilde{\xi}_{n}, \tilde{\xi}_{n}^{\prime}, q_{n}, \dot{q}_{n}\right), \frac{\partial \tilde{L}_{c}}{\partial \tilde{q}}\left(\tilde{\xi}_{n}, \tilde{\xi}_{n}^{\prime}, q_{n}, \dot{q}_{n}\right)\right)
$$

is bounded in $L^{1}\left([0, T], \mathbb{R}^{n+1}\right)$, thus its zero averaged primitive $P_{n} \in$ $W^{1,1}\left([0, T], \mathbb{R}^{n+1}\right)$ has a subsequence that is convergent in $L^{2}\left([0, T], \mathbb{R}^{n+1}\right)$. We suppose that

$$
P_{n} \stackrel{L^{2}}{\rightarrow} P .
$$

Since $\left\|d \mathcal{L}\left(\xi_{n}, q_{n}\right)\right\| \longrightarrow 0$, we have $\left\|d \tilde{\mathcal{L}}\left(\tilde{\xi}_{n}, q_{n}\right)_{\mid T \tilde{H}}\right\| \longrightarrow 0$, and the inequality

$$
\left|\int\left\langle\dot{P}_{n},(d \tilde{\xi}, d q)\right\rangle+\frac{\partial \tilde{L}_{c}}{\partial \nu} d \tilde{\xi}^{\prime}+\frac{\partial \tilde{L}_{c}}{\partial v} d \dot{q}\right| \leqslant \epsilon_{n}\|(d \tilde{\xi}, d q)\|_{H^{1}}
$$

holds for all variations $(d \tilde{\xi}, d q) \in H_{0}^{1}\left([0, T], \mathbb{R}^{n}\right) \subset T \tilde{H}$. The sequence

$$
m_{n}=\frac{1}{T} \int_{0}^{T}\left(\frac{\partial \tilde{L}_{c}}{\partial \nu}\left(\tilde{\xi}_{n}(t), \tilde{\xi}_{n}^{\prime}(t), q_{n}(t), \dot{q}_{n}(t)\right), \frac{\partial \tilde{L}_{c}}{\partial v}\left(\tilde{\xi}_{n}(t), \tilde{\xi}_{n}^{\prime}(t), q_{n}(t), \dot{q}_{n}(t)\right)\right) d t
$$

is bounded, and we can suppose taking a subsequence that it has a limit $m$. Integrating by parts in the inequality above yields

$$
\left\langle\left(\frac{\partial \tilde{L}_{c}}{\partial \nu}, \frac{\partial \tilde{L}_{c}}{\partial v}\right)-P_{n}-m_{n},\left(d \tilde{\xi}^{\prime}, d \dot{q}\right)\right\rangle_{L^{2}} \leqslant \epsilon_{n}\left\|\left(d \tilde{\xi}^{\prime}, d \dot{q}\right)\right\|_{L^{2}}
$$

and we obtain

$$
\left\|\left(\frac{\partial \tilde{L}_{c}}{\partial \nu}, \frac{\partial \tilde{L}_{c}}{\partial v}\right)-P_{n}-m_{n}\right\|_{L^{2}} \leqslant \epsilon_{n}
$$


We thus have

$$
\left(\frac{\partial \tilde{L}_{c}}{\partial \tilde{\xi}}\left(\tilde{\xi}_{n}, \tilde{\xi}_{n}^{\prime}, q_{n}, \dot{q}_{n}\right), \frac{\partial \tilde{L}_{c}}{\partial \tilde{q}}\left(\tilde{\xi}_{n}, \tilde{\xi}_{n}^{\prime}, q_{n}, \dot{q}_{n}\right)\right) \stackrel{L^{2}}{\longrightarrow} P-m
$$

and the sequence

$$
\left(\tilde{\xi}_{n}, \tilde{\xi}_{n}^{\prime}, q_{n}, \dot{q}_{n}\right)=\Upsilon_{c}^{-1}\left(\tilde{\xi}_{n}, \frac{\partial \tilde{L}_{c}}{\partial \tilde{\xi}}\left(\tilde{\xi}_{n}, \tilde{\xi}_{n}^{\prime}, q_{n}, \dot{q}_{n}\right), q_{n}, \frac{\partial \tilde{L}_{c}}{\partial \tilde{q}}\left(\tilde{\xi}_{n}, \tilde{\xi}_{n}^{\prime}, q_{n}, \dot{q}_{n}\right)\right)
$$

has a limit in $L^{2}$. The sequence $\left(\tilde{\xi}_{n}, q_{n}\right)$ thus has a limit in $H^{1}\left([0, T], \mathbb{R}^{n+1}\right)$, and the sequence $\left(\xi_{n}, q_{n}\right)=\rho^{-1}\left(\tilde{\xi}_{n}, q_{n}\right)$ has a limit in $H^{1}\left(\mathcal{O}_{c}\right) \times E_{T}$.

We now have to study the topology of the functional. Let us define a group $\Gamma$ of admissible deformations of $A_{T}$ :

Definition 3 A homeomorphism $h: A_{T} \longrightarrow A_{T}$ belongs to $\Gamma$ if and only if there exist a parameter $l \in(1,1+1 / \tau)$ and a continuous isotopy $k:[0,1] \times A_{T} \longrightarrow$ $A_{T}$ such that $k_{0}=I d, k_{1}=h$, and for all $t \in[0,1] k_{t}: A_{T} \longrightarrow A_{T}$ is a homeomorphism satisfying $k_{t}(\theta, q)=(\theta, q)$ when $\mathcal{Q}_{l}(q)+\int W(\partial \theta) \leqslant 0$.

For any compact subset $\sigma \subset \Lambda_{T}$ and any $h \in \Gamma$ we define the compact subset

$$
h . \sigma=P_{\Lambda}\left(h\left(\sigma \times E^{-}\right) \cap \Lambda \times E^{+}\right) \subset \Lambda .
$$

Lemma 8 (Intersection property ) Let $\Sigma$ be the family of compact subsets of $\Lambda_{T}$ defined in Section 5, Definition 1, we have

$$
\sigma \in \Sigma \text { and } h \in \Gamma \Longrightarrow h . \sigma \in \Sigma \text {. }
$$

Proof. Compare [20], Proposition 1. Let us consider the mapping

$$
\begin{aligned}
T_{s}: \sigma \times E^{-} & \longrightarrow E^{-} \\
(z, q) & \longmapsto T_{s}(z, q)=q-P^{-} \circ k_{s}(z, q),
\end{aligned}
$$

where $P^{-}: \Lambda_{T} \times E_{T} \longrightarrow E^{-}$is the projection associated with the splitting $E_{T}=E^{+} \oplus E^{-}$, and $k_{s}$ is the homotopy between $k_{0}=I d$ and $k_{1}=h$. Let us set

$$
F_{s}=\left\{(z, q) \in \sigma \times E^{-} / T_{s}(z, q)=q\right\}
$$

and

$$
I_{s}=k_{s}\left(\sigma \times E^{-}\right) \cap \Lambda \times E^{+}=k_{s}\left(F_{s}\right) .
$$

Both $I_{s}$ and $F_{s}$ contain $\left(\theta_{0}, 0\right)$. Since $\mathcal{Q}_{l}$ is negative definite on $E^{-}$and $\int W$ is bounded on $\sigma$, there is a $c>0$ such that $\mathcal{Q}_{l}(q)+\int W(\partial \theta)<0$ for all $\theta \in \sigma$ and $q \in E^{-}$satisfying $\|q\| \geqslant c$. As a consequence, the mapping $T_{s}$ satisfies

$-T_{0}=0$,

- $T_{s}(\theta, q)=0$ for all $q$ such that $\|q\| \geqslant c$ and all $s$,

- $T_{s}\left(\theta_{0}, q\right)=0$ for all $q$ and all $s$, 
and we can apply Dold's fixed point transfer, see [15] and [20], page 433, that asserts the injectivity of the morphism $P_{\Lambda}^{*}: H^{*}\left(\sigma, \theta_{0}\right) \longrightarrow H^{*}\left(F_{s},\left(\theta_{0}, 0\right)\right)$. We now take $s=1$ and have the commutative diagram

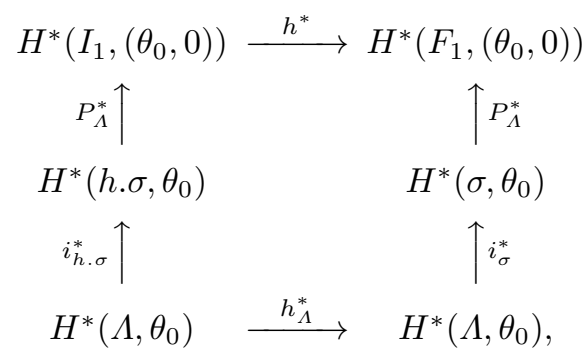

where $h_{\Lambda}^{*}$ is the isomorphism that makes the following diagram commute

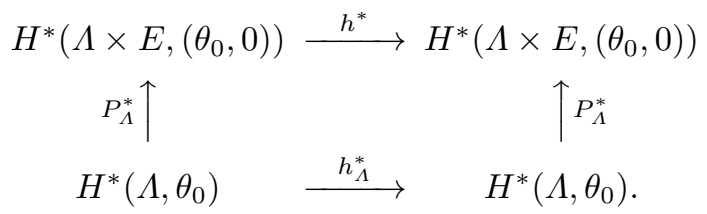

Coming back to the first diagram, we see that $i_{h . \sigma}^{*}$ can not be zero because $P_{\Lambda}^{*} \circ i_{\sigma}^{*} \circ h_{\Lambda}^{*}$ is nonzero.

For all $G$ satisfying [HR1-4] and all $l \in(1,1+1 / \tau)$ we define

$$
c_{T}^{G}(l)=\left.\inf _{\sigma \in \Sigma} \inf _{h \in \Gamma} \sup \mathcal{L}_{l}\right|_{h\left(\sigma \times E^{-}\right)}
$$

We have the estimate:

Lemma 9 If $G$ satisfies (2) then the inequality

$$
I_{T}(U) \leqslant c_{T}^{G}(l) \leqslant I_{T}(W)
$$

holds.

Proof. Since $G \longrightarrow c_{T}^{G}(l)$ is an increasing function this is an easy consequence of the following lemma.

Lemma 10 For all U satisfying [HU1-3], we have

$$
c_{T}^{U}(l)=I_{T}(U) .
$$

Proof. Recall that

$$
\begin{aligned}
c_{T}^{U}(l) & =\inf _{\sigma \in \Sigma} \inf _{h \in \Gamma} \sup _{(z, x) \in h\left(\sigma \times E^{-}\right)} \mathcal{Q}_{l}(x)+\mathcal{U}(z) \\
I_{T}(U) & =\inf _{\sigma \in \Sigma} \sup _{z \in \sigma}
\end{aligned}
$$

We can take $h=I d$ in the definition of $c$ to obtain

$$
c \leqslant \inf _{\sigma \in \Sigma} \sup _{\left(z, q^{-}\right) \in \sigma 99 \times E^{-}} \mathcal{Q}_{l}\left(q^{-}\right)+\mathcal{U}(z)=\inf _{\sigma \in \Sigma} \sup _{z \in \sigma} \mathcal{U}(z)=I .
$$


To obtain the other inequality, we apply Lemma 8 and get

$$
\sup _{(z, x) \in h\left(\sigma \times E^{-}\right)} \mathcal{Q}_{l}(x)+\mathcal{U}(z) \geqslant \sup _{z \in h . \sigma} \mathcal{U}(z) \geqslant I .
$$

We are now in a position to prove Proposition 2.

Proof of Proposition 2. First, notice that the third conclusion is a consequence of the two other ones since the only $T$ periodic solution of $L_{l}$ satisfying $\theta_{T} \equiv \theta_{0}$ is the constant curve $\left(\theta_{0}, 0\right)$, and has zero action, which is forbidden by the second conclusion since $c_{T}(l(T)) \geqslant I_{T}(U)>0$. Let us now choose $l(T)$. The function $l \longrightarrow c_{T}(l)$ is non-increasing thus almost everywhere differentiable. Moreover, the inequality

$$
\int_{1}^{1+1 / \tau} c_{T}^{\prime}(l) d l \geqslant I_{T}(U)-I_{T}(W)
$$

holds and we can choose an $l(T)$ in the interval $(1,1 / \tau)$ such that

$$
c^{\prime}=\left|c_{T}^{\prime}(l(T))\right| \leqslant \tau\left(I_{T}(W)-I_{T}(U)\right) .
$$

Let us set $c=c_{T}(l(T))$ and recall that

$$
\mathcal{L}_{l}(\theta, q)=\mathcal{L}(\theta, q)-a l^{2} \omega^{2}\|q\|_{2}^{2} .
$$

We shall prove that there exists a critical point $X_{T}=\left(\theta_{T}, q_{T}\right)$ of $\mathcal{L}_{l(T)}$ such that

$$
2 a \omega^{2}\left\|q_{T}\right\|_{2}^{2} \leqslant 1+c .^{\prime}
$$

We partially follow the presentation of [21] in the following. Arguing by contradiction we assume that there is no critical point of $L_{l(T)}$ at level $c$ satisfying $2 a \omega^{2}\left\|q_{T}\right\|_{2}^{2} \leqslant 1+c^{\prime}$. We can then find using a standard deformation argument an $\epsilon$ in the interval $(0, c / 2)$ and a homeomorphism $h_{0} \in \Gamma$ satisfying

$$
\mathcal{L}_{l(T)}\left(h_{0}(X)\right) \leqslant \mathcal{L}_{l(T)}(X)
$$

for all $X \in A_{T}$, and such that

$$
\mathcal{L}_{l(T)}\left(h_{0}(X)\right) \leqslant c-\epsilon
$$

for all $X=(\theta, q) \in A_{T}$ satisfying

$$
\mathcal{L}_{l(T)}(X) \leqslant c+\epsilon \quad \text { and } \quad 2 a \omega^{2}\|q\|_{2}^{2} \leqslant c^{\prime}+1 / 2 .
$$

Let $l_{n}$ be an increasing sequence converging to $l(T)$, and let $c_{n}=c_{T}\left(l_{n}\right)$ and $\mathcal{L}_{n}=\mathcal{L}_{l_{n}}$. We can choose $\sigma_{n} \in \Sigma$ and $h_{n} \in \Gamma$ such that

$$
\left.\sup \mathcal{L}_{n}\right|_{h_{n}\left(\sigma_{n} \times E^{-}\right)} \leqslant c_{n}+\left(l(T)-l_{n}\right) / 10 .
$$

When $n$ is large enough this implies

$$
\begin{aligned}
\left.\mathcal{L}_{l(T)}\right|_{h_{n}\left(\sigma_{n} \times E^{-}\right)} \leqslant\left.\mathcal{L}_{n}\right|_{h_{n}\left(\sigma_{n} \times E^{-}\right)} & \leqslant c_{n}+\left(l(T)-l_{n}\right) / 10 \\
& \leqslant c+\left(c^{\prime}+1 / 10\right)\left(l(T)-l_{n}\right)+\left(l(T)-l_{n}\right) / 10 \\
& \leqslant c+\left(c^{\prime}+1 / 5\right)\left(l(T)-l_{n}\right) .
\end{aligned}
$$


Take a loop $X=(\theta, q) \in h_{n}\left(\sigma_{n} \times E^{-}\right)$, either

$$
\mathcal{L}_{l(T)}(X) \leqslant c-\left(l(T)-l_{n}\right) / 5
$$

and

$$
\mathcal{L}_{l(T)}\left(h_{0}(X)\right) \leqslant c-\left(l(T)-l_{n}\right) / 5
$$

or

$$
\mathcal{L}_{l(T)} \geqslant c-\left(l(T)-l_{n}\right) / 5 .
$$

In the second case,

$$
\begin{aligned}
\left(l(T)^{2}-l_{n}^{2}\right) a \omega^{2}\|q\|^{2} & =\mathcal{L}_{n}(X)-\mathcal{L}_{l(T)}(X) \leqslant c+\left(l(T)-l_{n}\right)\left(c^{\prime}+1 / 5\right) \\
& -c+\left(l(T)-l_{n}\right) / 5 \leqslant\left(c^{\prime}+1 / 2\right)\left(l(T)-l_{n}\right),
\end{aligned}
$$

thus

$$
2 a \omega^{2}\|q\|^{2} \leqslant\left(l(T)+l_{n}\right) a \omega^{2}\|q\|^{2} \leqslant c^{\prime}+1 / 2,
$$

and we get

$$
\mathcal{L}_{l(T)}\left(h_{0}(X)\right) \leqslant c-\epsilon,
$$

when $n$ is large enough. We have seen that

$$
\mathcal{L}_{l(T)}\left(h_{0} \circ h_{n}\left(\sigma_{n} \times E^{-}\right)\right) \leqslant c-\left(l(T)-l_{n}\right) / 5,
$$

which is a contradiction since $h_{0} \circ h_{n} \in \Gamma$.

\section{References}

1. Arnold V. I.: Mathematical methods of classical mechanics, Springer (1978)

2. Arnold V. I., Kozlov V. V., Neishtadt A.I.: Mathematical Aspects of Classical and Celestial Mechanics, Encyclopaedia of Mathematical Sciences, Dynamical Systems III, Springer (1988)

3. Benci V., Giannoni F.: Homoclinic orbits on compact manifolds, J. Math. Anal. Appl. 157 (1991), 568-576

4. Bernard P.: Homoclinic orbits in families of hypersurfaces with hyperbolic periodic orbits, Jour. Diff. Eqn. 180 (2002), 427-452

5. Bernard P.: Recherche variationnelle d'orbites homoclines dans les systèmes dynamiques hamiltoniens, thèse, 2000

6. Bolotin S.V.: Libration motions of natural dynamical systems, Vestnik Mosk. Univ. Ser I Mat. 6 (1978), 72-77

7. Bolotin S.V.: The existence of homoclinic motions, Vestnik Mosk. Univ. Ser I Mat. Mekh. 6 (1983), 98-102

8. Bott R.: Lectures on Morse theory, old and new, BAMS 7 (1982), No. 2, 331-358

9. Buffoni B., Séré E.: A global condition for quasi-random behavior in a class of conservative systems, Comm. Pure and App. Math. 49 (1996), 285-305

10. Cielebak K.: Pseudoholomorphic curves and periodic orbits on cotangent bundles, J. Math. Pures Appl. (a) 73 (1994), 251-278

11. Cielebak K., Séré E.: Pseudoholomorphic curves and multiplicity of homolcinic orbits, Duke Math. J. 77 (1995), 483-518

12. Cielebak K., Séré E.: Pseudoholomorphic curves and the shadowing lemma, Duke Math. J. 98 (1999), 41-79 
13. Conley C. C.: On the ultimate behavior of orbits with respect to an unstable critical point I. oscillating, asymptotic and capture orbits, J. Diff. Eq. 5 (1969), 136-158

14. Coti Zelati V., Ekeland I., and Sere E.: A Variational Approach to Homoclinic Orbits in Hamiltonian Systems, Math. Ann. 288 (1990), 133-160

15. Dold A.: The fixed point transfer of fibre preserving maps, Math. Z. 148 (1976), 215-244

16. Devaney R. L.: Homoclinic orbits in hamiltonian systems, J. Diff. Eq. 21 (1976), 431438

17. Felmer P.: Heteroclinic orbits for spatially periodic hamiltonian systems, Ann. Inst. H. Poincaré, Anal. Non Linéaire 8 (1991), 477-497

18. Fenichel N.: Persistence and smoothness of invariant manifolds for flows, Ind. Univ. Math. Jour. 26 (1971), 193-225

19. Hirsch M. W., Pugh C. C., Shub M.: Invariant Manifolds, Lecture Notes in Math. 583 (1977), Springer

20. Hofer H., Viterbo C.: The Weinstein conjecture in cotangent bundles and related results, Ann. Scuola Normale Superiore di Pisa, Serie 515 (1988), 411-445

21. Jeanjean L.: On the existence of bounded PS sequences and applications to LandesmanLazer-type problem in $\mathbb{R}^{n}$, Proc. R. Soc. Edim., sect A, Math 129, No 4, (1999), 787-808

22. Klingenberg W.: Lectures on closed geodesics, Grundlehren der Math. Wiss. 230 (1978), Springer

23. Lerman L. M.: Hamiltonian systems with loops of a separatrix of a saddle-center, Selecta Math. Sov. 10 (1991), 297-306

24. Mawhin J., Willem M.: Critical point theory and Hamiltonian systems, App. Math. Sciences 105 (1989), Springer

25. Mielke A., Holmes P. and O'Reilly O.: Cascades of homoclinic orbits to, and chaos near, a Hamiltonian saddle-center, J. Dyn. Diff. Eq. 4 (1992), 95-126

26. Rabinowitz P. H.: Minimax methods in critical point theory with applications to differential equations, CBMS 65, AMS, Providence (1986)

27. Rabinovitz P.: Periodic and heteroclinic orbits for a periodic Hamiltonian system, Ann. Inst. H. Poincaré, Anal. Non Linéaire 6 (1989), 331-346

28. Ragazzo C. G.: Irregular dynamics and homoclinic orbits to hamiltonian saddle-centers, Comm. Pure Appl. Math. 49 (1996), 1-37

29. Struwe M.: Variational Methods, Springer, Second Edition, (1996)

30. Sullivan D.: Differential forms and the topology of manifolds, Proc. Int. Conf. on Manifolds and Related Topics in Topology, Tokyo (1973), 37-49 